\title{
Neural field model of binocular rivalry
}

waves

\section{Paul C. Bressloff \& Matthew A. Webber}

Journal of Computational

\section{Neuroscience}

ISSN 0929-5313

J Comput Neurosci

DOI 10.1007/s10827-011-0351y

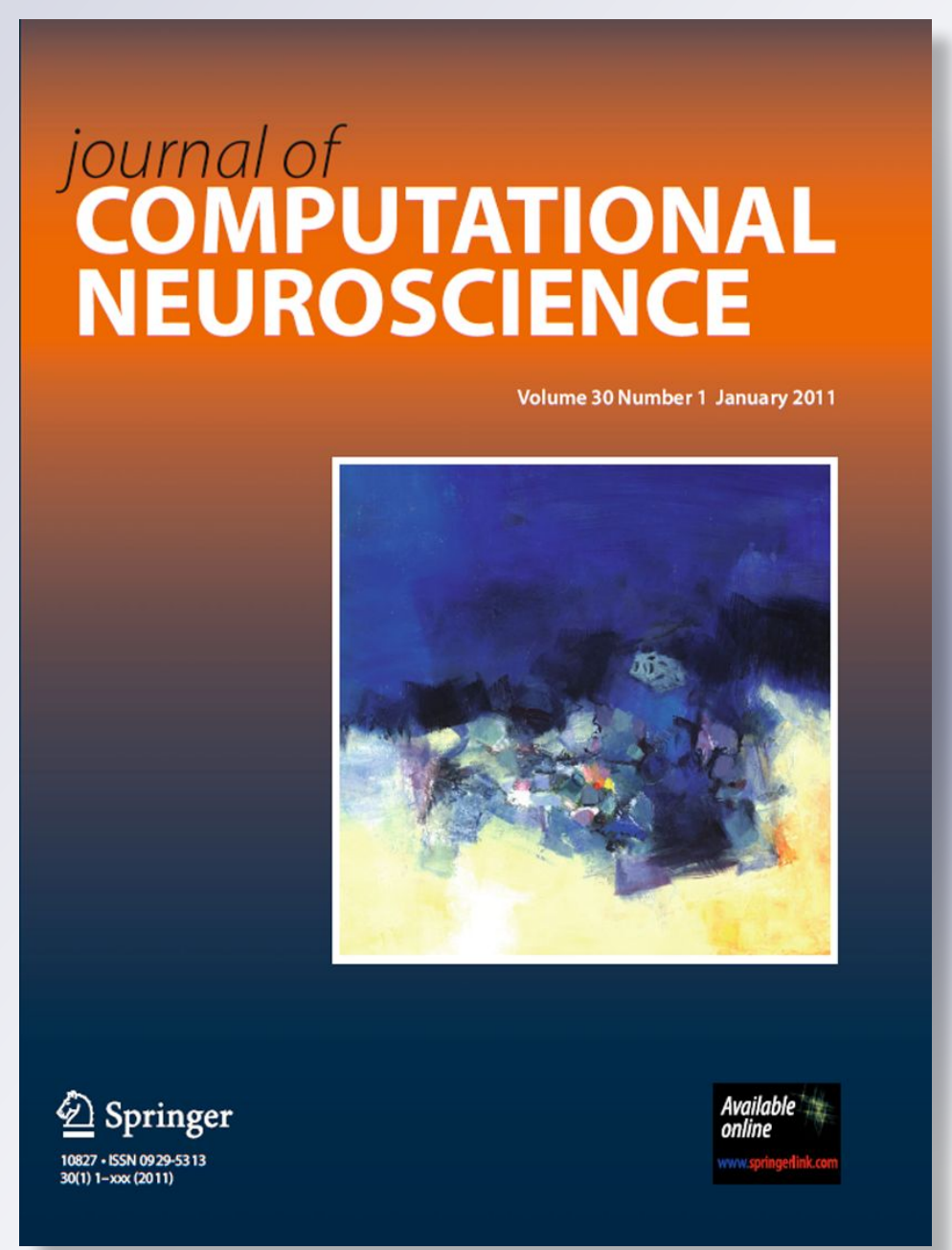

望 Springer 
Your article is protected by copyright and all rights are held exclusively by Springer Science+Business Media, LLC. This e-offprint is for personal use only and shall not be selfarchived in electronic repositories. If you wish to self-archive your work, please use the accepted author's version for posting to your own website or your institution's repository. You may further deposit the accepted author's version on a funder's repository at a funder's request, provided it is not made publicly available until 12 months after publication. 


\title{
Neural field model of binocular rivalry waves
}

\author{
Paul C. Bressloff • Matthew A. Webber
}

Received: 28 April 2011 / Revised: 20 June 2011 / Accepted: 22 June 2011

(C) Springer Science+Business Media, LLC 2011

\begin{abstract}
We present a neural field model of binocular rivalry waves in visual cortex. For each eye we consider a one-dimensional network of neurons that respond maximally to a particular feature of the corresponding image such as the orientation of a grating stimulus. Recurrent connections within each one-dimensional network are assumed to be excitatory, whereas connections between the two networks are inhibitory (crossinhibition). Slow adaptation is incorporated into the model by taking the network connections to exhibit synaptic depression. We derive an analytical expression for the speed of a binocular rivalry wave as a function of various neurophysiological parameters, and show how properties of the wave are consistent with the wavelike propagation of perceptual dominance observed in recent psychophysical experiments. In addition to providing an analytical framework for studying binocular rivalry waves, we show how neural field methods provide insights into the mechanisms underlying the generation of the waves. In particular, we highlight the important role of slow adaptation in providing a "symmetry breaking mechanism" that allows waves to propagate.
\end{abstract}

\section{Action Editor: Carson C. Chow}

P. C. Bressloff · M. A. Webber

Mathematical Institute, University of Oxford,

24-29 St. Giles', Oxford, OX1 3LB, UK

P. C. Bressloff $(\bowtie)$

Department of Mathematics, University of Utah,

Salt Lake City, UT 84112-0090, USA

e-mail: bressloff@math.utah.edu
Keywords Binocular rivalry • Neural fields • Synaptic depression $\cdot$ Cortical waves $\cdot$ Oscillations

\section{Introduction}

A number of phenomena in visual perception involve wave-like propagation dynamics. Examples include perceptual filling-in (De et al. 1998), migraine aura (Hadjikhani et al. 2001), expansion of illusory contours (Gold and Shubel 2006) and the line-motion illusion (Jancke et al. 2004). Another important example, which is the focus of this paper, is the wave-like propagation of perceptual dominance during binocular rivalry (Wilson et al. 2001; Lee et al. 2005; Kang et al. 2009, 2010). Binocular rivalry is the phenomenon where perception switches back and forth between different images presented to the two eyes. The resulting fluctuations in perceptual dominance and suppression provide a basis for non-invasive studies of the human visual system and the identification of possible neural mechanisms underlying conscious visual awareness (Blake 2001; Blake and Logothetis 2002). One way to observe and measure the speed of perceptual waves in psychophysical experiments (Wilson et al. 2001; Lee et al. 2005) is to take the rival images to be a lowcontrast radial grating presented to one eye and a highcontrast spiral grating presented to the other eye. Each image is restricted to an annular region of the visual field centered on the fixation point of the observer, thus effectively restricting wave propagation to the one dimension around the annulus. Switches in perceptual dominance can then be triggered using a brief rapid increase in stimulus contrast within a small region of the suppressed low-contrast grating. This induces a 
perceptual traveling wave in which the observer perceives the local dominance of the low-contrast image spreading around the annulus. The observer presses a key when the perceptual wave reaches a target area at a fixed distance from the trigger zone, and this determines the wave speed (Wilson et al. 2001; Lee et al. 2005). Since the rival images consist of oriented gratings, one might expect that primary visual cortex (V1) plays some role in the generation of binocular rivalry waves. Indeed, it has been shown using functional magnetic resonance imaging that there is a systematic correspondence between the spatiotemporal dynamics of activity in V1 and the time course of perceptual waves (Lee et al. 2005). However, it has not been established whether the waves originate in V1 or are evoked by feedback from extrastriate cortex.

Recently Kang et al. $(2009,2010)$ have developed a new psychophysical method for studying binocular rivalry waves that involves periodic perturbations of the rival images. An observer tracks rivalry within a small, central region of spatially extended rectangular grating patterns, while alternating contrast triggers are presented repetitively in the periphery of the rival patterns. The basic experimental set up is illustrated in Fig. 1. A number of interesting results have been obtained from these studies. First, over a range of trigger frequencies, the switching between rival percepts within the central regions is entrained to the triggering events. Moreover, the optimal triggering frequency depends on the natural frequency of spontaneous switching (in the absence of triggers). Second, the latency between triggering event and perceptual switching increases approximately linearly with the distance between the triggering site and the central region being tracked by the observer, consistent with the propagation of a traveling front. Third, the speed of the traveling wave across observers covaries with the spontaneous switching rate.

In this paper we analyze the existence and stability of binocular rivalry waves in a continuum neural field
Fig. 1 Schematic diagram illustrating experimental protocol used to study binocular rivalry waves (Kang et al. 2009). High contrast triggers are presented periodically in antiphase within the upper extended region of one grating pattern and within the lower region of the rival pattern. Subject simply reports perceptual alternations in rival dominance within the central monitoring region indicated by the horizontal black lines on each pattern. The monitoring region is a distance $\Delta d$ from the trigger region, which can be adjusted. If $\Delta t$ is the latency between the triggering event and the subsequent observation of a perceptual switch, then the speed $c$ of the wave is given by the slope of the plot $\Delta d=c \Delta t$

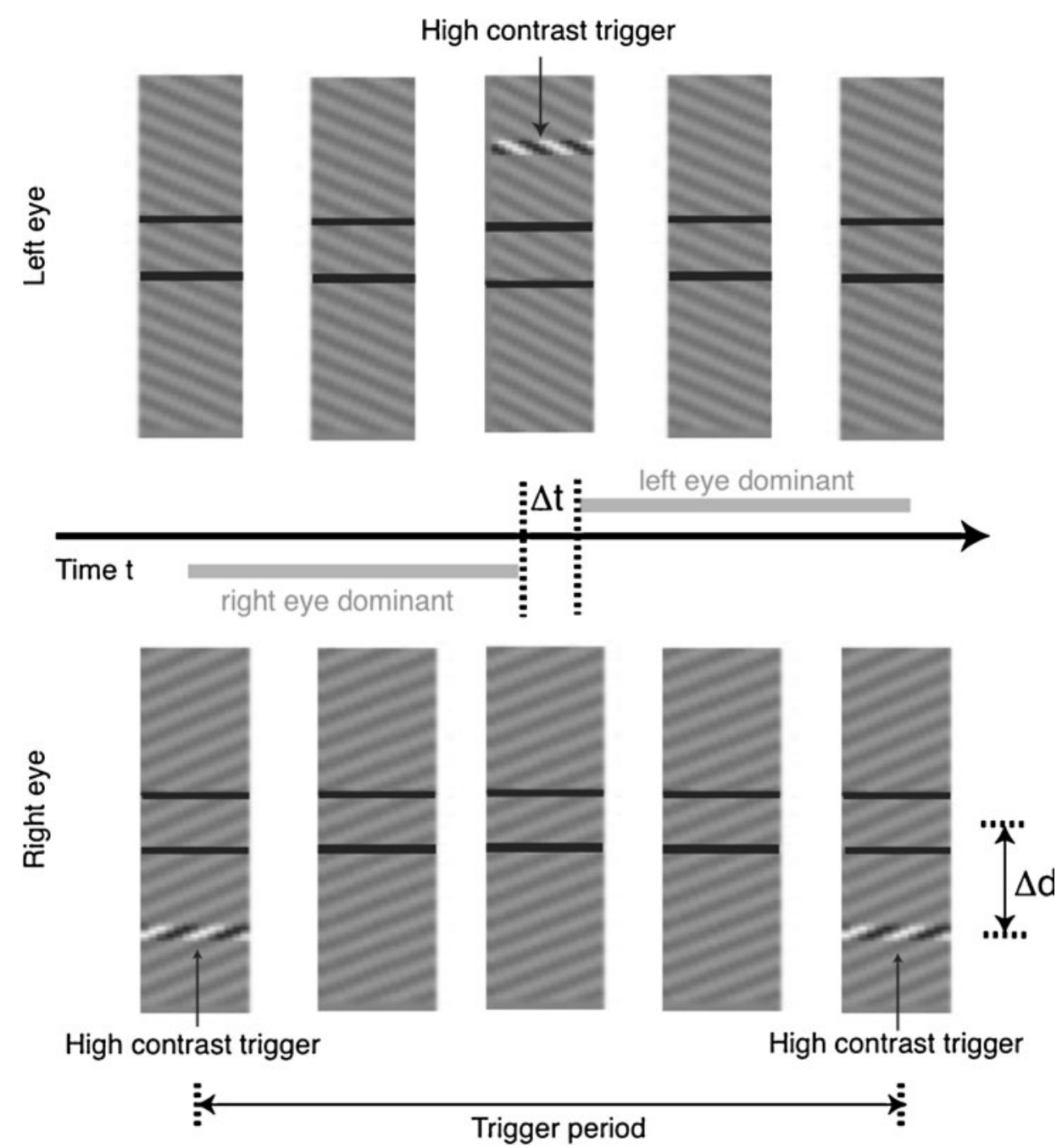

Trigger period 
model of visual cortex. We derive an analytical expression for the wave speed as a function of neurophysiological parameters, and use this to show how our model reproduces various experimental results found by Kang et al. $(2009,2010)$, in particular, the observation that wave speed covaries with the natural alternation rate. Our model is essentially a continuum version of a previous computational model of rivalry waves based on a discrete two layer neural network (Wilson et al. 2001; Kang et al. 2010). The two layers represent cortical neurons responsive to one or more features of the image presented to the left and right eye respectively. Such features could include the orientation of a spatially extended grating stimulus or distinguish between spiral and radial annulus patterns. In the discrete computational model, connections within a layer were taken to be excitatory, whereas between layers, neurons mutually inhibited each other via a set of local interneurons. Moreover, in order to allow switching between the dominant and suppressed populations, the excitatory neurons were assumed to exhibit some form of slow spike frequency adaptation. In our neural field version of this model, we consider an alternative form of slow adaptation based on depressing synapses. In addition to providing an analytical framework for studying binocular rivalry waves, we show how neural field methods provide insights into the mechanisms underlying the generation of the waves. In particular, we highlight the important role of slow adaptation in providing a "symmetry breaking mechanism" that allows fronts to propagate.

\section{The model}

In order to model binocular rivalry waves in the presence of oriented grating stimuli (Wilson et al. 2001; Kang et al. 2009, 2010), it is useful to review some of the stimulus response properties of neurons in the primary visual cortex (V1) (Hubel and Wiesel 1962, 1977). First, each neuron in V1 responds to light stimuli in a restricted region of the visual field called its classical receptive field; stimuli outside a neuron's receptive field do not directly affect its activity. Second, most neurons in V1 respond preferentially to stimuli targeting either the left or right eye, which is known as ocular dominance. It has been suggested that neurons with different ocular dominance may inhibit one another if they have nearby receptive fields (Katz et al. 1989). Third, most neurons in V1 are tuned to respond maximally when a stimulus of a particular orientation is in their receptive field (Hubel and Wiesel 1962; Blasdel 1992). This is known as a neuron's orientation preference, and the neuron will not respond if a stimulus is sufficiently different from its preferred orientation. Finally, multielectrode recordings and optical imaging have established that at each point in the visual field there exists a corresponding set of neurons spanning the entire spectrum of orientation preferences that are packed together as a unit in V1, known as a hypercolumn (Hubel and Wiesel 1977; Tootell et al. 1998; Blasdel 1992). Within each hypercolumn, neurons with sufficiently similar orientations tend to excite each other whereas those with sufficiently different orientations inhibit each other, and this serves to sharpen a particular neuron's orientation preference (Ben-Yishai et al. 1995; Ferster and Miller 2000). Moreover, anatomical evidence suggests that inter-hypercolumn connections link neurons with similar orientation preferences (Sincich and Blasdel 2001; Angelucci et al. 2002). The functional relationship between stimulus feature preferences and synaptic connections within V1 thus suggests that V1 is a likely substrate of simple examples of binocular rivalry such as those involving sinusoidal grating stimuli (Sterzer et al. 2009).

Binocular rivalry waves consist of two basic components: the switching between rivalrous left/right eye states and the propagation of the switched state across a region of cortex. Let us first focus on the switching mechanism by neglecting spatial effects. Suppose that distinct oriented grating patterns are presented to the two eyes such as those shown in Fig. 1. This induces rivalry due to the combination of orientation specific and ocular dominant cross-inhibition in V1 (BenYishai et al. 1995; Sincich and Blasdel 2001; Blake and Logothetis 2002). During left eye stimulus dominance, it is assumed that a group of the left eye neurons that respond to 135 degree orientations, say, are firing persistently, while right eye neurons are suppressed by cross-inhibitory connections. Following this, some slow adaptive process such as synaptic depression or spike frequency adaptation causes a switch so that right eye neurons tuned to 45 degree orientations fire persistently, suppressing the left eye neurons. The cycle of left eye neural dominance along with right eye neural suppression followed by right eye neural dominance along with left eye neural suppression can then repeat itself in the form of rivalrous oscillations. This basic model of reciprocal inhibition paired with a slow adaptive process has often been used to phenomenologically model the neural substrate of binocular rivalry oscillations (Fox and Rasche 1969; Skinner et al. 1994; Wilson et al. 2001; Laing and Chow 2002; Taylor et al. 2002; Shpiro et al. 2007, 2009; Kilpatrick and Bressloff 2010).

In order to take into account the propagation of activity seen in binocular rivalry waves (Wilson et al. 
2001; Kang et al. 2009, 2010), we will consider a continuum neural field model. Several previous studies have modeled the spontaneous switching between rivalrous oriented stimuli in terms of a pair of ring networks (neural fields on a periodic domain) with slow adaptation and cross-inhibition, representing a pair of hypercolumns for the left and right eyes, respectively (Laing and Chow 2002; Kilpatrick and Bressloff 2010). In these models, the rivalrous states consist of stationary activity bumps coding for either the left or right eye stimuli. (Rivalry effects in a spatially extended model have also been examined in a prior study by Loxley and Robinson (Loxley and Robinson 2009), in which rivalrous stimuli are presented to a single one-dimensional network). However, these models were not used to study binocular rivalry waves and, as formulated, were on the wrong spatial scale since they only considered short-range spatial scales comparable to a single hypercolumn. One way to proceed would be to construct a more general coupled ring model along the lines of Bressloff et al. (2001) and Bressloff and Cowan (2002), in which two sets of hypercolumns (ring networks) are distributed along a pair of lines corresponding to the left and right eyes, respectively, such that along each line the synaptic weights can be decomposed into shortrange intra-columnar interactions and long-range intercolumnar interactions, together with cross-inhibitory connections between the left and right eye networks. In this paper, we will consider a simpler network architecture in which we neglect the internal structure of a hypercolumn. That is, for each eye we consider a one-dimensional network of neurons whose orientation preference coincides with the orientation of the corresponding grating stimulus, which is a reasonable first approximation from the perspective of the experiments conducted by Kang et al. $(2009,2010)$. Recurrent connections within each one-dimensional network are assumed to be excitatory, whereas connections between the two networks are inhibitory (cross-inhibition). Slow adaptation is incorporated into the model by taking the network connections to exhibit synaptic depression along the lines of Kilpatrick and Bressloff (2010). The basic architecture is shown schematically in Fig. 2. Note that a similar network architecture was previously considered in a computational model of binocular rivalry waves (Wilson et al. 2001; Kang et al. 2010), in which cross inhibition was mediated explicitly by interneurons and, rather than including depressing synapses, the excitatory neurons were taken to exhibit spike frequency adaptation. The most significant difference between our approach and the previous computational approaches (besides considering synaptic depression rather than spike frequency adaptation) is that the latter considers a discrete neural network rather than a continuum neural field. As we will establish in this paper, one advantage of neural field theory is that analytical methods can be used to derive conditions for the existence and stability of traveling waves, and to derive formulae relating the wave speed to various neurophysiological parameters.

Let $u(x, t)$ and $v(x, t)$ denote the activity of the left and right eye networks at position $x \in \mathbb{R}$ at time $t$. The

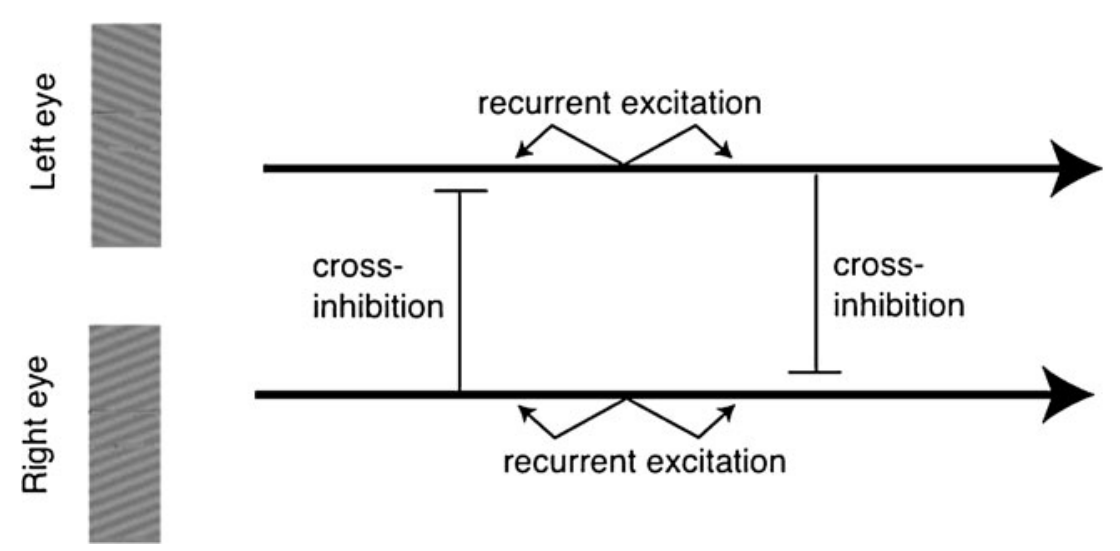

Fig. 2 Schematic diagram of network architecture consisting of two one-dimensional neural fields. Suppose that the left eye is shown a 135 degree grating and the right eye is shown a 45 degree grating. Then one of the neural fields represents the activity of neurons tuned to 135 degree orientations in the left eye, whilst the other neural field represents the activity of neurons tuned to 45 degree orientations in the right eye. Recurrent connections within each one-dimensional network are assumed to be excitatory, whereas connections between the two networks are inhibitory (cross-inhibition). The excitatory and inhibitory weight distributions are taken to be Gaussians. Slow adaptation is incorporated into the model by taking the network connections to exhibit synaptic depression 
associated neural field equations are taken to be of the form

$$
\begin{aligned}
\tau \frac{\partial u(x, t)}{\partial t}= & -u(x, t)+I_{u}(x, t) \\
& \left.+\int_{-\infty}^{\infty} w_{e}\left(x-x^{\prime}\right) q_{u}\left(x^{\prime}, t\right) f\left(u\left(x^{\prime}, t\right)\right)\right) d x^{\prime} \\
& \left.-\int_{-\infty}^{\infty} w_{i}\left(x-x^{\prime}\right) q_{v}\left(x^{\prime}, t\right) f\left(v\left(x^{\prime}, t\right)\right)\right) d x^{\prime}
\end{aligned}
$$

$$
\tau_{s} \frac{\partial q_{u}(x, t)}{\partial t}=1-q_{u}(x, t)-\beta q_{u}(x, t) f(u(x, t)),
$$

and

$$
\begin{aligned}
\tau \frac{\partial v(x, t)}{\partial t}= & -v(x, t)+I_{v}(x, t) \\
& \left.+\int_{-\infty}^{\infty} w_{e}\left(x-x^{\prime}\right) q_{v}\left(x^{\prime}, t\right) f\left(v\left(x^{\prime}, t\right)\right)\right) d x^{\prime} \\
& \left.-\int_{-\infty}^{\infty} w_{i}\left(x-x^{\prime}\right) q_{u}\left(x^{\prime}, t\right) f\left(u\left(x^{\prime}, t\right)\right)\right) d x^{\prime},
\end{aligned}
$$

$$
\tau_{s} \frac{\partial q_{v}(x, t)}{\partial t}=1-q_{v}(x, t)-\beta q_{v}(x, t) f(v(x, t)) .
$$

The nonlinear function $f$ represents the mean firing rate of a local population and is usually taken to be a smooth, bounded monotonic function such as a sigmoid

$$
f(u)=\frac{1}{1+\mathrm{e}^{-\eta(u-\kappa)}},
$$

with gain $\eta$ and threshold $\kappa$. However, in order to derive explicit traveling wave solutions, it will be convenient to consider the high gain limit $\eta \rightarrow \infty$ of Eq. (2.3) such that $f$ becomes a Heaviside function (Amari 1977; Pinto and Ermentrout 2001; Coombes and Owen 2004)

$f(u)=H(u-\kappa)=\left\{\begin{array}{l}0 \text { if } u<\kappa \\ 1 \text { if } u>\kappa .\end{array}\right.$

The distribution $w_{e}$ of excitatory connections between neurons of the same eye preference and the distribution of cross-inhibitory connections between neurons of the opposite eye preference are both taken to be Gaussians:

$$
w_{e}(r)=\frac{a_{e}}{\sqrt{2 \pi \sigma_{e}^{2}}} e^{-\frac{r^{2}}{2 \sigma_{e}^{2}}}, \quad w_{i}(r)=\frac{a_{i}}{\sqrt{2 \pi \sigma_{i}^{2}}} e^{-\frac{r^{2}}{2 \sigma_{i}^{2}}}
$$

We assume that at baseline excitatory connections are longer range than inhibitory connections, ${ }^{1} \sigma_{e}>\sigma_{i}$ and fix length scales by setting $\sigma_{e}=2$ and $\sigma_{i}=1$ at baseline. We expect excitatory connections to span a cortical hypercolumn so that $\sigma_{e}$ will be of the order $200 \mu \mathrm{m}$. We also fix the temporal scale of the network by setting the membrane time constant $\tau=1$; the membrane time constant is typically around $10 \mathrm{~ms}$. Depressing synapses are incorporated into the model in the form of the presynaptic scaling factors $q_{u}, q_{v}$ evolving according to Eqs. (2.1b) and (2.2b). These scaling factors can be interpreted as a measure of available presynaptic resources, which are depleted at a rate proportional to $\beta q f$ (Tsodyks et al. 1998; Tabak et al. 2000; Bart et al. 2005), and are recovered on a timescale specified by the constant $\tau_{s}$. Specifically, we will study the effect of slow short term synaptic depression (experimentally shown to recover over 5-10 s (Varela et al. 1997; Chance et al. 1998)). Slow short term synaptic depression has been implicated as a mechanism for contrast adaptation in V1, due to its comparable recovery timescale of 5$10 \mathrm{~s}$ (Varela et al. 1997). Thus, there is evidence for participation of this slower depression in processes of V1 in addition to faster short term synaptic depression, which recovers on timescales of roughly $200-800 \mathrm{~ms}$ (Abbott et al. 1997; Varela et al. 1997). Finally, we take $I_{u}, I_{v}$ to represent the effective strength of the left and right eye stimuli, respectively. Recall that we are only modeling neurons whose orientation preference coincides with the corresponding stimulus orientation so that we can take the unperturbed network to have constant homogeneous inputs. (The induction of traveling waves requires locally perturbing one of the inputs).

\section{Travelling waves}

3.1 Non-depressing synapses $(\beta=0)$

In our simplified model, we interpret the binocular rivalry wave seen in the experiments of Kang et al.

\footnotetext{
${ }^{1}$ From a mathematical perspective, it would also be possible to generate waves when the cross-inhibition is longer range than the excitatory connections, see Fig. 4. Which form is more realistic from the biological perspective depends on which classes of neurons are being taken into account by the neural field model. For example, in visual cortex it is known that excitatory pyramidal cells make both local synaptic contacts as well as longerrange horizontal connections. However, the latter innervate both excitatory and local inhibitory neurons so they could have a net inhibitory effect, thus providing a possible source of longrange inhibition; whether long-range connections generate net excitation or net inhibition also depends on stimulus conditions (Lund et al. 2003).
} 
$(2009,2010)$ as a traveling wave front solution of the neural field equations (2.1) and (2.2), in which a high activity state invades the suppressed left eye network, say, whilst retreating from the dominant right eye network. Let us begin by ignoring the effects of synaptic depression, that is, we set $\beta=0$ and $q_{u}=q_{v} \equiv 1$. We also assume that both inputs have the same strength or contrast so that $I_{u}=I_{v}=I$. We then obtain the reduced system of equations (for $\tau=1$ and $f$ given by the Heaviside (2.4))

$$
\begin{aligned}
\frac{\partial u}{\partial t}+u= & \int_{-\infty}^{\infty} w_{e}\left(x-x^{\prime}\right) H\left(u\left(x^{\prime}, t\right)-\kappa\right) d x^{\prime} \\
& -\int_{-\infty}^{\infty} w_{i}\left(x-x^{\prime}\right) H\left(v\left(x^{\prime}, t\right)-\kappa\right) d x^{\prime}+I,
\end{aligned}
$$

and

$$
\begin{aligned}
\frac{\partial v}{\partial t}+v= & \int_{-\infty}^{\infty} w_{e}\left(x-x^{\prime}\right) H\left(v\left(x^{\prime}, t\right)-\kappa\right) d x^{\prime} \\
& -\int_{-\infty}^{\infty} w_{i}\left(x-x^{\prime}\right) H\left(u\left(x^{\prime}, t\right)-\kappa\right) d x^{\prime}+I .
\end{aligned}
$$

Given that the differential operator $D=\frac{\partial}{\partial t}+1$ has a Green's function given by $G(t, s)=\eta(t-s)$ with

$\eta(t)= \begin{cases}0 & t<0 \\ e^{-t} & t>0\end{cases}$

we can re-write the above equations in purely integral form:

$$
\begin{aligned}
u(x, t)=I & +\int_{0}^{\infty} \eta(s) G_{e}[u](x, t-s) d s \\
& -\int_{0}^{\infty} \eta(s) G_{i}[v](x, t-s) d s \\
v(x, t)=I+ & \int_{0}^{\infty} \eta(s) G_{e}[v](x, t-s) d s \\
& -\int_{0}^{\infty} \eta(s) G_{i}[u](x, t-s) d s
\end{aligned}
$$

where

$$
G_{p}[u](x, t)=\int_{-\infty}^{\infty} w_{p}(y) H(u(x-y, t)-\kappa) d y
$$

for $p=e, i$.

Homogeneous fixed point solutions $\left(U^{*}, V^{*}\right)$ of Eqs. (3.3) and (3.4) satisfy the pair of equations

$$
\begin{aligned}
& U^{*}=a_{e} H\left(U^{*}-\kappa\right)-a_{i} H\left(V^{*}-\kappa\right)+I \\
& V^{*}=a_{e} H\left(V^{*}-\kappa\right)-a_{i} H\left(U^{*}-\kappa\right)+I,
\end{aligned}
$$

where we have used the normalization of the Gaussians (2.5)

$\int_{-\infty}^{\infty} w_{e}(x) d x=a_{e}, \quad \int_{-\infty}^{\infty} w_{i}(x) d x=a_{i}$.

There are a maximum of four possible fixed point solutions, all of which are stable. First, there is the off state $U^{*}=V^{*}=I$, which occurs when $I<\kappa$, that is, the input is not strong enough to directly activate either population. Second there is the on-state or fusion state $U^{*}=V^{*}=a_{e}-a_{i}+I$, which occurs when $I>\kappa+a_{i}-$ $a_{e}$. This case is more likely when recurrent excitation is strong or cross-inhibition is weak. Finally, there are two winner-take-all (WTA) states in which one population dominates the other: the left eye dominant state $\left(U^{*}, V^{*}\right)=\mathbf{X}_{L} \equiv\left(a_{e}+I, I-a_{i}\right)$ and the right eye dominant state $\left(U^{*}, V^{*}\right)=\mathbf{X}_{R} \equiv\left(I-a_{i}, a_{e}+I\right)$. These states exist when $I>\kappa-a_{e}$ and $I<\kappa+a_{i}$.

Let us now consider a traveling wave front solution of the form

$u(x, t)=U(x-c t), \quad v(x, t)=V(x-c t)$

where $c$ is the wave speed and $\xi=x-c t$ is a traveling wave coordinate. We assume that

$(U(\xi), V(\xi)) \rightarrow \mathbf{X}_{L}$ as $\xi \rightarrow-\infty$,

$(U(\xi), V(\xi)) \rightarrow \mathbf{X}_{R}$ as $\xi \rightarrow \infty$

with $U(\xi)$ a monotonically decreasing function of $\xi$ and $V(\xi)$ a monotonically increasing function of $\xi$. It follows that if $c>0$ then the wavefront represents a solution in which activity invades a supressed left eye network and retreats from a dominant right eye network. Substituting the traveling front solution into Eqs. (3.3) and (3.4) gives

$$
\begin{aligned}
U(\xi)=I & +\int_{0}^{\infty} \eta(s) \widehat{G}_{e}[U](\xi+c s) d s \\
& -\int_{0}^{\infty} \eta(s) \widehat{G}_{i}[V](\xi+c s) d s \\
V(\xi)=I & +\int_{0}^{\infty} \eta(s) \widehat{G}_{e}[V](\xi+c s) d s \\
& -\int_{0}^{\infty} \eta(s) \widehat{G}_{i}[U](\xi+c s) d s .
\end{aligned}
$$

where

$\widehat{G}_{p}[U](\xi)=\int_{-\infty}^{\infty} w_{p}(y) H(U(\xi-y)-\kappa) d y, p=e, i$

Given the asymptotic behavior of the solution and the requirements of monotonicity, we see that $U(\xi)$ 
and $V(\xi)$ each cross threshold at a single location, which may be different for the two eyes. Exploiting translation invariance we take $U(0)=\kappa$ and $V\left(\xi_{0}\right)=$ $\kappa$. These threshold crossing conditions imply that the above equations simplify further according to

$U(\xi)=\int_{0}^{\infty} \eta(s)\left[\int_{\xi+c s}^{\infty} w_{e}(y) d y-\int_{-\infty}^{\xi-\xi_{0}+c s} w_{i}(y) d y\right] d s+I$

$V(\xi)=\int_{0}^{\infty} \eta(s)\left[\int_{-\infty}^{\xi-\xi_{0}+c s} w_{e}(y) d y-\int_{\xi+c s}^{\infty} w_{i}(y) d y\right] d s+I$.

It is convenient to introduce the function

$\Psi_{\xi_{0}}(z)=\int_{z}^{\infty} w_{e}(y) d y-\int_{-\infty}^{z-\xi_{0}} w_{i}(y) d y$.

Exploiting the fact that the Gaussian weight distributions $w_{e}(y)$ and $w_{i}(y)$ are even functions of $y$, it can be seen that

$\Psi_{\xi_{0}}\left(\xi_{0}-z\right)=\int_{-\infty}^{z-\xi_{0}} w_{e}(y) d y-\int_{z}^{\infty} w_{i}(y) d y$,

so that Eqs. (3.7) and (3.8) can be rewritten in the more compact form

$U(\xi)=\int_{0}^{\infty} \eta(s) \Psi_{\xi_{0}}(\xi+c s) d s+I$
$V(\xi)=\int_{0}^{\infty} \eta(s) \Psi_{\xi_{0}}\left(\xi_{0}-\xi-c s\right)+I$.

Finally, imposing the threshold conditions $U(0)=$ $V\left(\xi_{0}\right)=\kappa$ gives

$\kappa=\int_{0}^{\infty} \eta(s) \Psi_{\xi_{0}}(c s) d s+I$,

$\kappa=\int_{0}^{\infty} \eta(s) \Psi_{\xi_{0}}(-c s) d s+I$.

It is now straightforward to show that a traveling front solution cannot exist for the neural field model without adaptation given by Eqs. (3.1) and (3.2). For subtracting Eq. (3.13) from Eq. (3.12) implies that

$\int_{0}^{\infty} \eta(s)\left[\Psi_{\xi_{0}}(c s)-\Psi_{\xi_{0}}(-c s)\right] d s=0$,

which does not have a solution for any $c, \xi_{0}$ such that $c \neq 0$. The latter follows from Eq. (3.9), which shows that for $c \neq 0$,

$$
\begin{aligned}
& c\left[\Psi_{\xi_{0}}(c s)-\Psi_{\xi_{0}}(-c s)\right] \\
& =-c \int_{-c s}^{c s} w_{e}(y) d y-c \int_{-c s-\xi_{0}}^{c s-\xi_{0}} w_{i}(y) d y<0
\end{aligned}
$$

whereas $\eta(s)>0$ for all $s \in[0, \infty)$. The non-existence of traveling fronts is consistent with the observation that in the absence of any cross inhibition $\left(w_{i} \equiv 0\right)$, the system reduces to two independent one-dimensional neural fields with excitatory connections. In order to construct a front solution that simultaneously invades one network whilst retreating from the other we would require a one dimensional excitatory neural field to be able to support a pair of counter-propagating front solutions with speeds $\pm c$, which is not possible (Amari 1977). Therefore, some mechanism must be introduced that breaks the exchange symmetry of the two onedimensional networks. One way to break the symmetry would be to take the input strengths of the left and right eye stimuli to be sufficiently different, $I_{u} \neq I_{v}$. However, this case can be excluded, since rivalry waves are still observed experimentally when both inputs are of equal strength. Moreover, a traveling front could only travel in one direction, depending on the sign of $I_{L}-I_{R}$. Therefore, we consider an alternative mechanism based on slow synaptic depression.

\subsection{Slow synaptic depression $\left(\beta>0, \tau_{s} \gg 1\right)$}

Let us now consider the full neural field model with depressing synaptic connections given by Eqs. (2.1) and (2.2) with $f(u)=H(u-\kappa)$. As in the case $\beta=0$, there are four possible homogeneous fixed points corresponding to an off-state, a fusion state and two WTA states, and all are stable. Denoting a homogeneous fixed point by $\left(U^{*}, V^{*}, Q_{u}^{*}, Q_{v}^{*}\right)$, we have

$U^{*}=Q_{u}^{*} a_{e} H\left(U^{*}-\kappa\right)-Q_{v}^{*} a_{i} H\left(V^{*}-\kappa\right)+I$

$Q_{u}^{*}=\frac{1}{1+\beta H\left(U^{*}-\kappa\right)}$

$V^{*}=Q_{v}^{*} a_{e} H\left(V^{*}-\kappa\right)-Q_{u}^{*} a_{i} H\left(U^{*}-\kappa\right)+I$

$Q_{v}^{*}=\frac{1}{1+\beta H\left(V^{*}-\kappa\right)}$

with $a_{e}, a_{i}$ given by Eq. (3.6). Hence, the fusion state is now

$$
\begin{aligned}
& \left(U^{*}, V^{*}\right)=\left(\frac{a_{e}-a_{i}}{1+\beta}+I, \frac{a_{e}-a_{i}}{1+\beta}+I\right), \\
& \left(Q_{u}^{*}, Q_{v}^{*}\right)=\left(\frac{1}{1+\beta}, \frac{1}{1+\beta}\right),
\end{aligned}
$$

and occurs when $I>\kappa-\left(a_{e}-a_{i}\right) /(1+\beta)$. This case is more likely for very strong depression ( $\beta$ large), since cross inhibition will be weak, or when the local 
connections are strong and excitation-dominated. The left eye dominant WTA state now takes the form

$$
\begin{aligned}
& \left(U^{*}, V^{*}\right)=\left(\frac{a_{e}}{1+\beta}+I, I-\frac{a_{i}}{1+\beta}\right), \\
& \left(Q_{u}^{*}, Q_{v}^{*}\right)=\left(\frac{1}{1+\beta}, 1\right)
\end{aligned}
$$

whereas the right eye dominant WTA state becomes

$$
\begin{aligned}
& \left(U^{*}, V^{*}\right)=\left(I-\frac{a_{i}}{1+\beta}, \frac{a_{e}}{1+\beta}+I\right), \\
& \left(Q_{u}^{*}, Q_{v}^{*}\right)=\left(1, \frac{1}{1+\beta}\right)
\end{aligned}
$$

The WTA states exist provided that

$$
I>\kappa-\frac{a_{e}}{1+\beta}, \quad I<\kappa+\frac{a_{i}}{1+\beta}
$$

This will occur in the presence of weak depression $(\beta$ small) and strong cross-inhibition such that depression cannot exhaust the dominant hold one population has on the other. Previously, we have shown that Eqs. (2.1) and (2.2) also support homogeneous limit cycle oscillations in which there is periodic switching between left and right eye dominance consistent with binocular rivalry (Kilpatrick and Bressloff 2010). Since all the fixed points are stable, it follows that such oscillations cannot arise via a standard Hopf bifurcation. Indeed, we find bistable regimes where a rivalry state coexists with a fusion state as illustrated in Fig. 3. (Such behavior persists in the case of smooth sigmoid firing rate

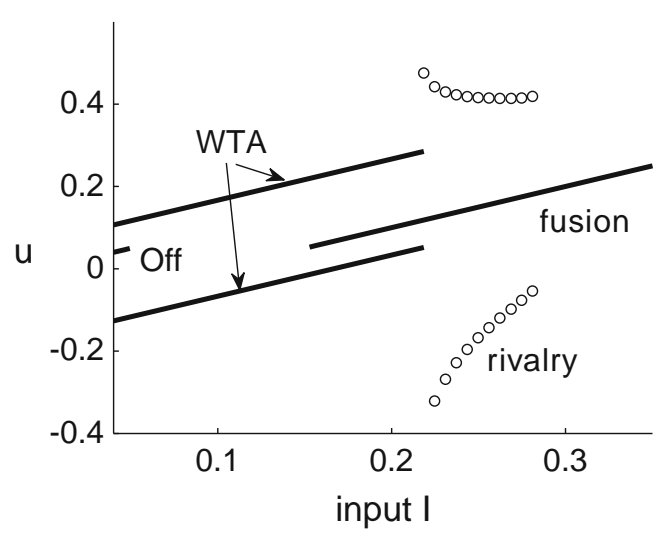

Fig. 3 Bifurcation diagram showing homogeneous solutions for the left population activity $u$ as a function of the input amplitude I. Solid lines represent stable states, whereas circles represent the maximum and minimum of rivalrous oscillations. It can be seen that there are regions of off/WTA bistability, WTA/fusion bistability, and fusion/rivalry bistability. Parameters are $\tau_{s}=500$, $\kappa=0.05, \beta=5, a_{e}=0.4$ and $a_{i}=1$ functions, at least for sufficiently high gain (Kilpatrick and Bressloff 2010)).

Suppose that the full system given by Eqs. (2.1) and (2.2) is initially in a stable right eye dominated WTA state, and is then perturbed away from this state by introducing a propagating front that generates a switch from right to left eye dominance. We further assume that over a finite spatial domain of interest the time taken for the wave front to propagate is much smaller than the relaxation time $\tau_{s}$ of synaptic depression. Thus, to a first approximation we can ignore the dynamics of the depression variables and assume that they are constant, that is, $\left(q_{u}(x, t), q_{v}(x, t)\right)=\left(Q_{u}, Q_{v}\right)$ with $Q_{u}=1$ and $Q_{v}=(1+\beta)^{-1}$. A similar adiabatic approximation can also be made if the network is in a binocular rivalry state, provided that (a) the duration of wave propagation is short compared to the natural switching period and (b) the induction of the wave does not occur close to the point at which spontaneous switching occurs. In this case $Q_{u}$ and $Q_{v}$ will not be given by the WTA solution, but we can assume that $Q_{u} \neq Q_{v}$. Under the adiabatic approximation, we obtain a slightly modified version of Eqs. (3.3) and (3.4) given by

$$
\begin{aligned}
u(x, t)= & I+Q_{u} \int_{0}^{\infty} \eta(s) G_{e}[u](x, t-s) d s \\
& -Q_{v} \int_{0}^{\infty} \eta(s) G_{i}[v](x, t-s) d s
\end{aligned}
$$

$$
\begin{aligned}
v(x, t)= & I+Q_{v} \int_{0}^{\infty} \eta(s) G_{e}[v](x, t-s) d s \\
& -Q_{u} \int_{0}^{\infty} \eta(s) G_{i}[u](x, t-s) d s .
\end{aligned}
$$

with $G_{e, i}$ given by Eq. (3.5). The analysis of the existence of a traveling wave front solution proceeds along identical lines to Section 3.1, except that now the asymptotic states take the form

$\mathbf{X}_{L}=\left(Q_{u} a_{e}+I, I-Q_{v} a_{i}\right)$,

$\mathbf{X}_{R}=\left(I-Q_{u} a_{i}, Q_{v} a_{e}+I\right)$.

We thus obtain the following modified threshold conditions:

$\kappa=\int_{0}^{\infty} \eta(s) \Psi_{\xi_{0}}(c s) d s+I$,
$\kappa=\int_{0}^{\infty} \eta(s) \Phi_{\xi_{0}}(-c s) d s+I$, 
with $\Psi$ and $\Phi$ defined by

$\Psi_{\xi_{0}}(z)=Q_{u} \int_{z}^{\infty} w_{e}(y) d y-Q_{v} \int_{-\infty}^{z-\xi_{0}} w_{i}(y) d y$.

$\Phi_{\xi_{0}}(z)=Q_{v} \int_{z}^{\infty} w_{e}(y) d y-Q_{u} \int_{-\infty}^{z-\xi_{0}} w_{i}(y) d y$.

It can be seen that synaptic depression breaks the symmetry of the consistency equations, and this will allow us to find solutions for $c, \xi_{0}$ and thus establish the existence of traveling front solutions.

\subsection{Calculation of wave speed}

In order to establish the existence of a wave speed $c$ and a threshold crossing point $\xi_{0}$, define the functions

$F_{1}\left(c, \xi_{0}\right)=\int_{0}^{\infty} \eta(s) \Psi_{\xi_{0}}(c s) d s$

and

$F_{2}\left(c, \xi_{0}\right)=\int_{0}^{\infty} \eta(s) \Phi_{\xi_{0}}(-c s) d s$.

Evaluating the integrals using Eqs. (2.5), (3.22), (3.23) and $\eta(s)=\mathrm{e}^{-s}$ gives (for $c>0$ )

$$
\begin{array}{r}
F_{1}\left(c, \xi_{0}\right)=\frac{a_{e} Q_{u}}{2}\left(1-e^{\frac{\sigma_{e}^{2}}{2 \tau_{f}^{2} c^{2}}} \operatorname{Erfc}\left[\frac{\sigma_{e}}{\sqrt{2} \tau_{f} c}\right]\right) \\
-\frac{a_{i} Q_{v}}{2}\left(\operatorname{Erfc}\left[\frac{\xi_{0}}{\sqrt{2} \sigma_{i}}\right]-e^{\frac{\sigma_{i}^{2}-2 \tau_{f} c \xi_{0}}{2 \tau_{f} c^{2}}}\right. \\
\left.\times \operatorname{Erfc}\left[\frac{\sigma_{i}^{2}-\tau_{f} c \xi_{0}}{\sqrt{2} \sigma_{i} \tau_{f} c}\right]\right) \\
F_{2}\left(c, \xi_{0}\right)=\frac{a_{e} Q_{v}}{2}\left(1+e^{\frac{\sigma_{e}^{2}}{2 \tau_{f}^{2} c^{2}}} \operatorname{Erfc}\left[\frac{\sigma_{e}}{\sqrt{2} \tau_{f} c}\right]\right) \\
-\frac{a_{i} Q_{u}}{2}\left(\operatorname{Erfc}\left[\frac{\xi_{0}}{\sqrt{2} \sigma_{i}}\right]-e^{\frac{\sigma_{i}^{2}+2 \xi_{0} \tau_{f} c}{2 \tau_{f}^{2} c^{2}}}\right. \\
\left.\times \operatorname{Erfc}\left[\frac{\sigma_{i}^{2}+\xi_{0} \tau_{f} c}{\sqrt{2} \sigma_{i} \tau_{f} c}\right]\right)
\end{array}
$$

where

$$
\operatorname{Erfc}(x)=\frac{2}{\sqrt{\pi}} \int_{x}^{\infty} \mathrm{e}^{-t^{2}} d t
$$

is the complementary error function. Subtracting Eq. (3.21) from Eq. (3.20), we then have the implicit equation

$$
G\left(c, \xi_{0}\right) \equiv F_{1}\left(c, \xi_{0}\right)-F_{2}\left(c, \xi_{0}\right)=0 .
$$

It is straightforward to show that for fixed $\xi_{0}$,

$\lim _{c \rightarrow \infty} G\left(c, \xi_{0}\right)>0, \quad \lim _{c \rightarrow-\infty} G\left(c, \xi_{0}\right)<0$.

Hence, the intermediate value theorem guarantees at least one solution $c=c\left(\xi_{0}\right)$, which is differentiable by the implicit function theorem. If $Q_{u}=Q_{v}$, then $F_{1}\left(0, \xi_{0}\right)=F_{2}\left(0, \xi_{0}\right)$ and the only point where $G$ vanishes is at $c=0$. On the other hand, if $Q_{v} \neq Q_{u}$ then $G\left(0, \xi_{0}\right) \neq 0$ for all finite $\xi_{0}$ so that $c\left(\xi_{0}\right) \neq 0$. Given a solution $c=c\left(\xi_{0}\right)$ of Eq. (3.28), the existence of a traveling wavefront solution reduces to the single threshold condition

$\kappa=F_{1}\left(c\left(\xi_{0}\right), \xi_{0}\right)+I$

We find that there exists a unique traveling front solution for a range of values of $\kappa$.

In Figs. 4 and 5, we plot wave speed as a function of various model parameters. We choose baseline parameter values such that spontaneous oscillations and traveling fronts co-exist, as found experimentally (Kang et al. 2009, 2010). The model wave speed is of the order $c=1$ in dimensionless units, that is, $c=\sigma_{e} / 2 \tau$ where $\sigma_{e}$ is the range of excitation and $\tau$ is the membrane time constant. In the psychophysical experiments of Kang et al. (2009, 2010), it was found that binocular rivalry waves took approximately $0.8 \mathrm{~s}$ to traverse 2 degrees of the visual field. The magnification factor in humans throughout the foveal region is approximately $0.4 \mathrm{~cm} / \mathrm{deg}$, which corresponds to $0.8 \mathrm{~cm}$ of cortex. Hence the wave speed we would expect to see in cortex based on the psychophysical experiments is approximately $10 \mathrm{~mm} / \mathrm{s}$. This is consistent with our analytical results, if we take $\sigma_{e}$ to be of the order $200 \mu \mathrm{m}$ and $\tau$ to be of the order $10 \mathrm{~ms}$. Figure 4 shows that increasing the strength of excitation (cross-inhibition) increases (decreases) the speed of the wave, whereas Fig. 5(a) shows that the speed of the wave decreases as the threshold $\kappa$ increases. However, these parameters cannot be manipulated in psychophysical experiments. On the other hand, it is possible to vary the contrast of the sinusoidal grating stimuli. For example, Kang et al. $(2009,2010)$ showed that increasing the stimulus contrast increases wave speed. This is consistent with Fig. 5(b), which plots wave speed as a function of input 

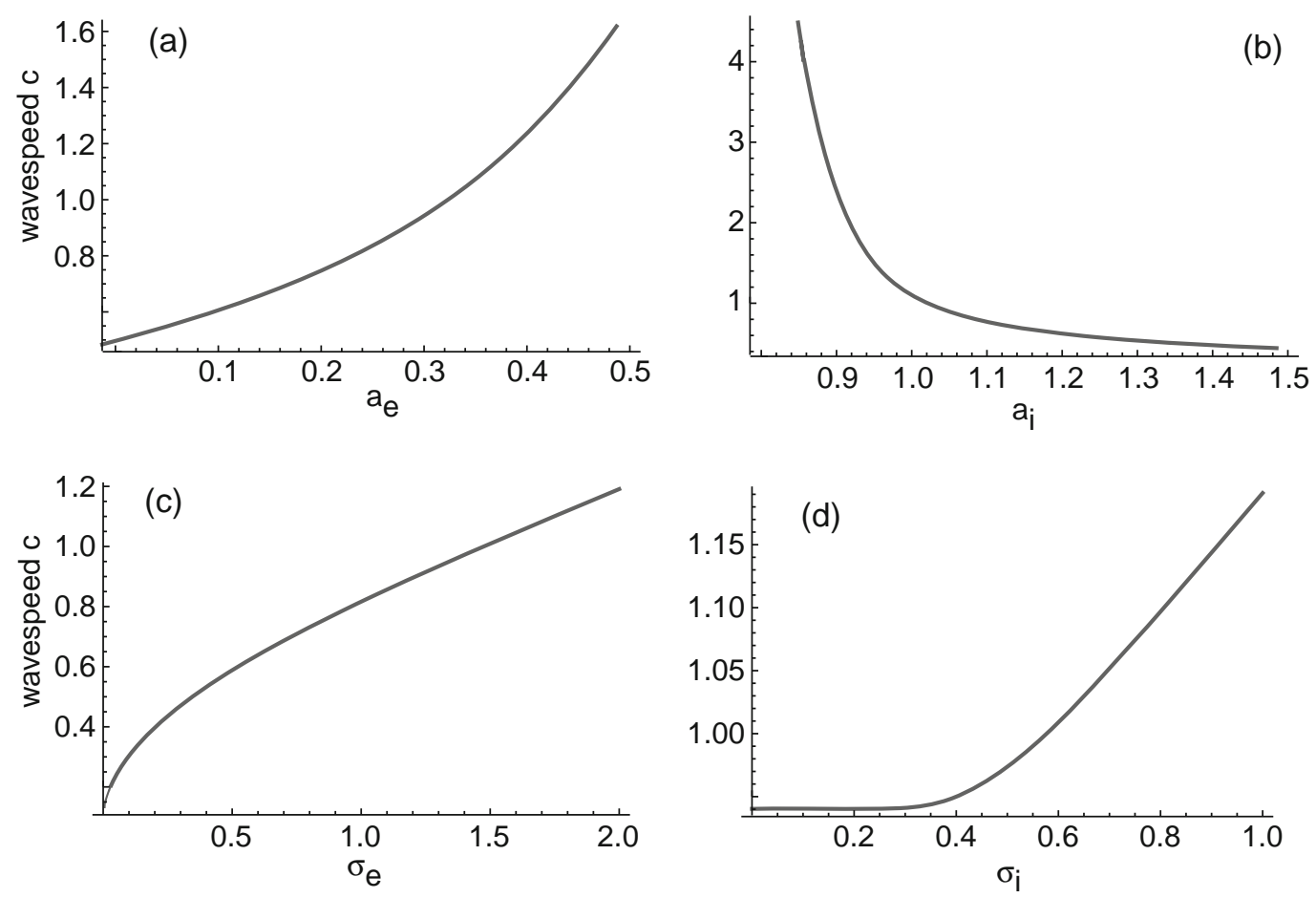

Fig. 4 Plot of wave speed $c$ as a function of various weight parameters: (a, b) the amplitudes $a_{e}, a_{i}$ and $(\mathbf{c}, \mathbf{d})$ the length constants $\sigma_{e}, \sigma_{i}$. The default parameters are taken to be $a_{i}=$ $1, a_{e}=0.4, \sigma_{i}=1, \sigma_{e}=2, \beta=5, \kappa=0.05, I=0.24, Q_{u}=0.42$,
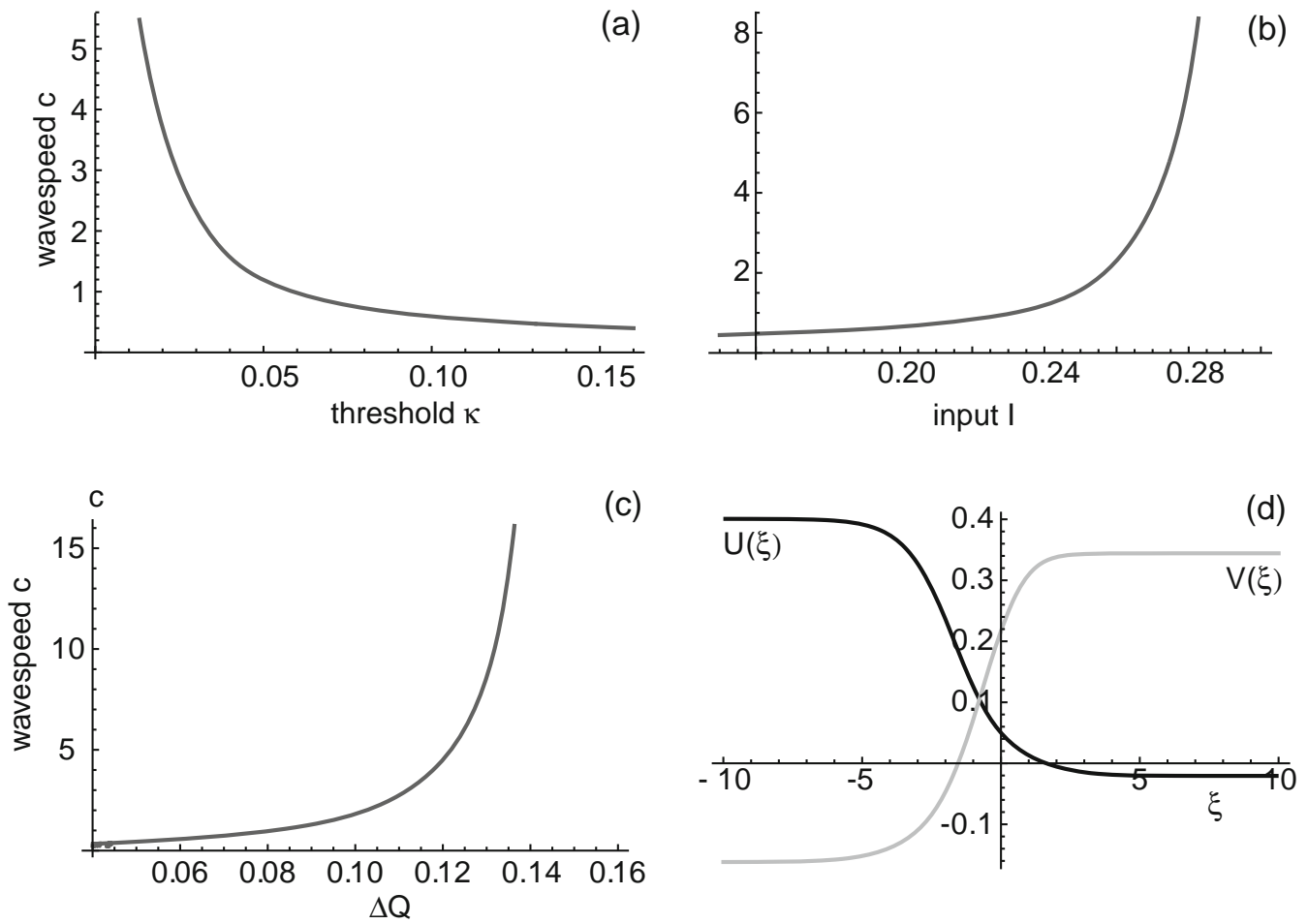

Fig. 5 (a-c) Plot of wave speed $c$ as a function of (a) the threshold $\kappa$, (b) the external input strength $I$ and (c) $\Delta Q$ where $Q_{u}=$ $Q_{0}+\Delta Q, Q_{v}=Q_{0}-\Delta Q$ with $Q_{0}=0.335$. (d) Plot of rightmoving traveling front solution in which a high activity state

$Q_{v}=0.25$ and the corresponding wave speed is $c=1.2$. For this set of parameters, the network operates in a regime that supports both traveling waves and homogeneous oscillatory solutions, that is, spontaneous switching occurs in the absence of traveling waves

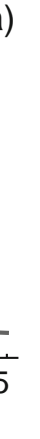

(c)

invades the suppressed left eye network whilst retreating from the dominant right eye network. Parameters are at the baseline values of Fig. 4 unless specified otherwise 
amplitude I. Another variable that could be manipulated is the time at which a rivalry wave is induced relative to the phase of the limit cycle representing spontaneous rivalry oscillations. In our model, under the adiabatic approximation, this would correspond to changing the values of $Q_{u}$ and $Q_{v}$. In Fig. 5(c) we plot wave speed as a function of $\Delta Q=\left(Q_{u}-Q_{v}\right) / 2$ with $Q_{u}+Q_{v}$ fixed. It can be seen that $c$ increases with $\Delta Q$, which is analogous to inducing a wave closer to the point at which spontaneous switching would occur (see also Section 4). Note that in the experimental protocol of Kang et al. (2009, 2010), see Fig. 1, the wave speed is determined after averaging with respect to the switching events induced by a sequence of periodic trigger stimuli rather than a single triggering event. In terms of our model, this would correspond to averaging the wave speed with respect to a range of values of $Q_{u}$ and $Q_{v}$, since spontaneous binocular rivalry oscillations are actually noisy. Hence, the dependence of wave speed on the timing of the inducing stimuli relative to the phase of spontaneous oscillations is effectively reduced. However, Kang et al. did find that there is an optimal trigger period for inducing reliable switching that depends on the natural period of spontaneous oscillations. It is likely that this phase-locking phenomenon reflects some residual dependence of wave propagation on the relative timing of the trigger stimuli. Finally, a typical example of a wavefront profile is shown in Fig. 5(d). Note that the symmetry breaking mechanism necessary to support wave propagation is reflected by the fact that $\lim _{\xi \rightarrow-\infty} U(\xi)>\lim _{\xi \rightarrow \infty} V(\xi)$. That is, the initial activity in the dominant right eye is depressed compared to the final activity in the dominant left eye (under the adiabatic approximation).

\subsection{Wave stability}

In order to determine wave stability, we linearize Eqs. (3.18) and (3.19) around the traveling wave solution $(U(\xi), V(\xi))$ with $Q_{u}, Q_{v}$ fixed. Writing $u(x, t)=$ $U(\xi)+r(\xi) e^{\lambda t}$ and $v(x, t)=V(\xi)+p(\xi) e^{\lambda t}$, we obtain the linear system

$$
\begin{aligned}
r(\xi)= & Q_{u} \int_{0}^{\infty} \eta(s) \int_{-\infty}^{\infty} w_{e}(y) r(\xi+c s-y) e^{-\lambda s} \\
& \times \delta(U(\xi+c s-y)-\kappa) d y d s \\
& -Q_{v} \int_{0}^{\infty} \eta(s) \int_{-\infty}^{\infty} w_{i}(y) p(\xi+c s-y) e^{-\lambda s} \\
& \times \delta(V(\xi+c s-y)-\kappa) d y d s
\end{aligned}
$$

$$
\begin{aligned}
p(\xi)= & Q_{v} \int_{0}^{\infty} \eta(s) \int_{-\infty}^{\infty} w_{e}(y) p(\xi+c s-y) e^{-\lambda s} \\
& \times \delta(V(\xi+c s-y)-\kappa) d y d s \\
& -Q_{u} \int_{0}^{\infty} \eta(s) \int_{-\infty}^{\infty} w_{i}(y) r(\xi+c s-y) e^{-\lambda s} \\
& \times \delta(U(\xi+c s-y)-\kappa) d y d s
\end{aligned}
$$

where $\delta(U-\kappa)$ denotes the Dirac delta function. Using the threshold conditions $U(0)=\kappa, V\left(\xi_{0}\right)=\kappa$ and a property of Dirac delta functions, we have

$$
\begin{aligned}
\delta(U(\xi)-\kappa) & =\frac{\delta(\xi)}{\left|U^{\prime}(0)\right|}, \\
\delta(V(\xi)-\kappa) & =\frac{\delta\left(\xi-\xi_{0}\right)}{\left|V^{\prime}\left(\xi_{0}\right)\right|}
\end{aligned}
$$

Hence,

$$
\begin{aligned}
r(\xi)= & Q_{u} \int_{0}^{\infty} \eta(s) w_{e}(\xi+c s) \frac{r(0)}{\left|U^{\prime}(0)\right|} e^{-\lambda s} d s \\
& -Q_{v} \int_{0}^{\infty} \eta(s) w_{i}\left(\xi+c s-\xi_{0}\right) \frac{p\left(\xi_{0}\right)}{\left|V^{\prime}\left(\xi_{0}\right)\right|} e^{-\lambda s} d s
\end{aligned}
$$

$$
\begin{aligned}
p(\xi)= & Q_{v} \int_{0}^{\infty} \eta(s) w_{e}\left(\xi+c s-\xi_{0}\right) \frac{p\left(\xi_{0}\right)}{\left|V^{\prime}\left(\xi_{0}\right)\right|} e^{-\lambda s} d s \\
& -Q_{u} \int_{0}^{\infty} \eta(s) w_{i}(\xi+c s) \frac{r(0)}{\left|U^{\prime}(0)\right|} e^{-\lambda s} d s .
\end{aligned}
$$

Introducing an appropriate norm on the space of functions $(r(\xi), p(\xi))$, the linear equations (3.31) and (3.32) generate a well defined spectral problem. In particular, the discrete spectrum of eigenvalues $\lambda$ is obtained by finding solutions $r(\xi)$ and $p(\xi)$ that decay sufficiently fast as $|\xi| \rightarrow \infty$-the rate of decay is determined by the weight functions $w_{e}, w_{i}$. These eigensolutions can be found by setting $\xi=0$ in Eq. (3.31) and $\xi=\xi_{0}$ in Eq. (3.32), and solving the resulting self-consistency equations for $r(0)$ and $p\left(\xi_{0}\right)$ :

$$
\left(\begin{array}{c}
r(0) \\
p\left(\xi_{0}\right)
\end{array}\right)=\left(\begin{array}{cc}
\frac{Q_{u} \widehat{W}_{e}(\lambda, 0)}{\left|U^{\prime}(\lambda)\right|} & -\frac{Q_{v} \widehat{W}_{i}\left(\lambda,-\xi_{0}\right)}{\left|V^{\prime}\left(\xi_{0}\right)\right|} \\
-\frac{Q_{u} \widehat{W}_{i}\left(\lambda, \xi_{0}\right)}{\left|U^{\prime}(0)\right|} & \frac{Q_{v} \widehat{W}_{e}(\lambda, 0)}{\left|V^{\prime}\left(\xi_{0}\right)\right|}
\end{array}\right)\left(\begin{array}{c}
r(0) \\
p\left(\xi_{0}\right)
\end{array}\right)
$$

where

$$
\widehat{W}_{p}(\lambda, \xi)=\int_{0}^{\infty} \eta(s) w_{p}(c s+\xi) e^{-\lambda s} d s
$$


for $p=e, i$. The self-consistency equation has a nontrivial solution provided that $\lambda$ is a zero of the function

$E(\lambda)=\operatorname{det}\left(\begin{array}{cc}\frac{Q_{u} \widehat{W}_{e}(\lambda, 0)}{\left|U^{\prime}(0)\right|}-1 & -\frac{Q_{v} \widehat{W}_{i}\left(\lambda,-\xi_{0}\right)}{\left|V^{\prime}\left(\xi_{0}\right)\right|} \\ -\frac{Q_{u} \widehat{W}_{i}\left(\lambda, \xi_{0}\right)}{\left|U^{\prime}(0)\right|} & \frac{Q_{v} \widehat{W}_{e}(\lambda, 0)}{\left|V^{\prime}\left(\xi_{0}\right)\right|}-1\end{array}\right)$

We identify $E(\lambda)$ with the so-called Evans function of the traveling wave solution (Zhang 2003; Coombes and Owen 2004; Sandstede 2007). That is, the complex number $\lambda$ is an eigenvalue of the linear system if and only if $E(\lambda)=0$. Moreover, the algebraic multiplicity of each eigenvalue is equal to the order of the corresponding zero of the Evans function. It can be shown that $E(\lambda)$ is analytic in $\lambda$ and thus normal numeric root finding may be used to find $\lambda$. The wave will be linearly stable if all non-zero eigenvalues $\lambda$ have negative real part and $\lambda=0$ is a simple eigenvalue. The existence of a zero eigenvalue reflects translation invariance of the dynamical system given by Eqs. (3.18) and (3.19). (Note that there is also a continuous part of the spectrum, but this always lies in the left-half complex $\lambda$-plane and thus does not contribute to any wave instabilities (Coombes and Owen 2004; Sandstede 2007)). Numerically plotting

(a)

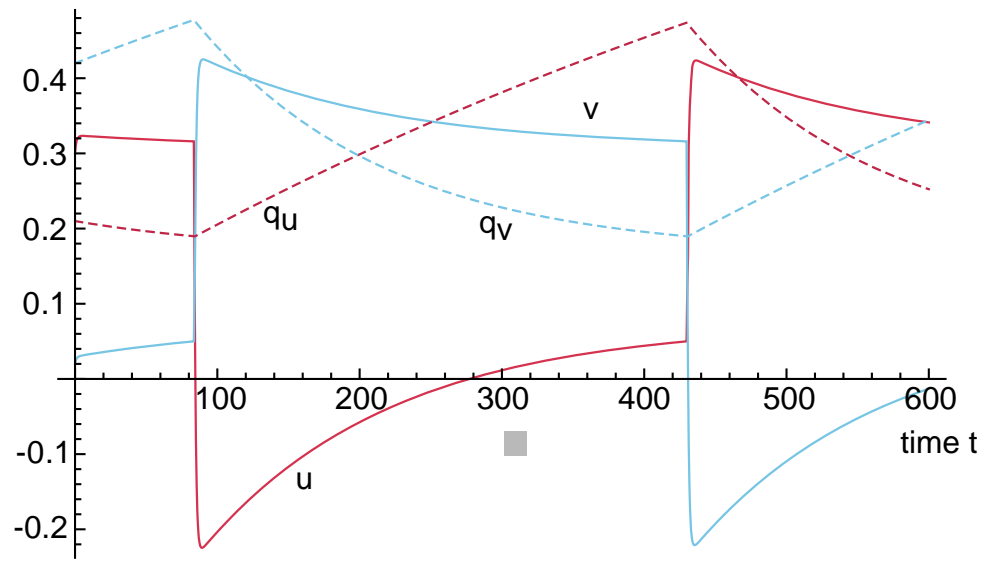

(b)

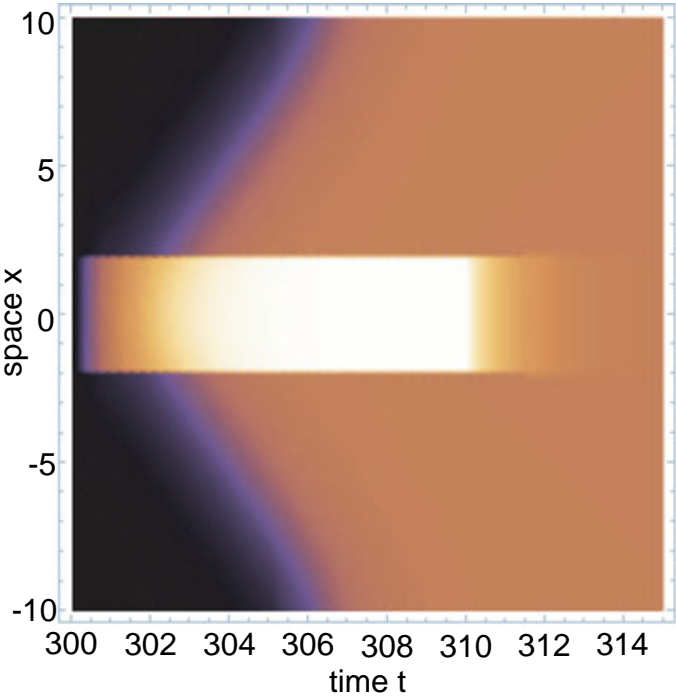

(c)

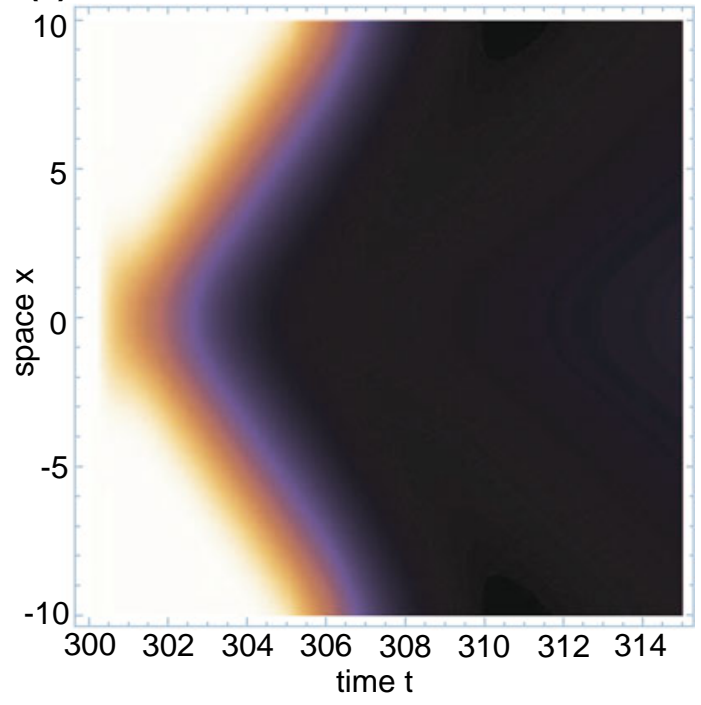

time $t_{0}=300$, which involves a temporary increase in the input strength $I_{u}$ from 0.24 to 0.74 within the domain $-2 \leq x \leq 2$. (The duration of the trigger stimulus is also indicated by the grey bar in the unperturbed trace of part (a)). Lighter (darker) colors indicate higher (lower) activity values. The mean wave speed of the front (calculated numerically)) is $c \sim 2$. This is in good agreement with our analytical results based on taking fixed depression variables $Q_{u}=q_{u}\left(t_{0}\right), Q_{v}=q_{v}\left(t_{0}\right)$ 
(a)

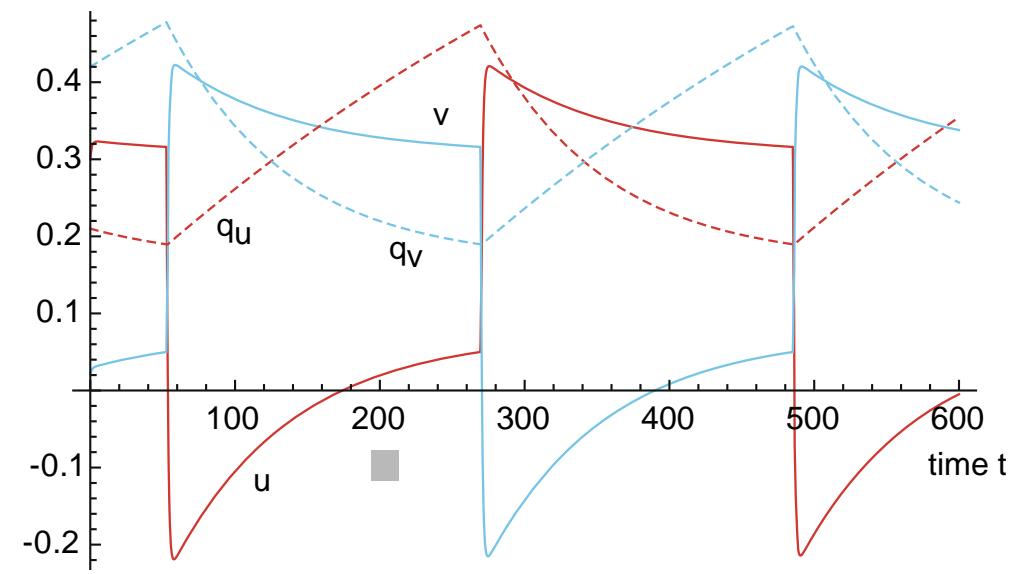

(b)

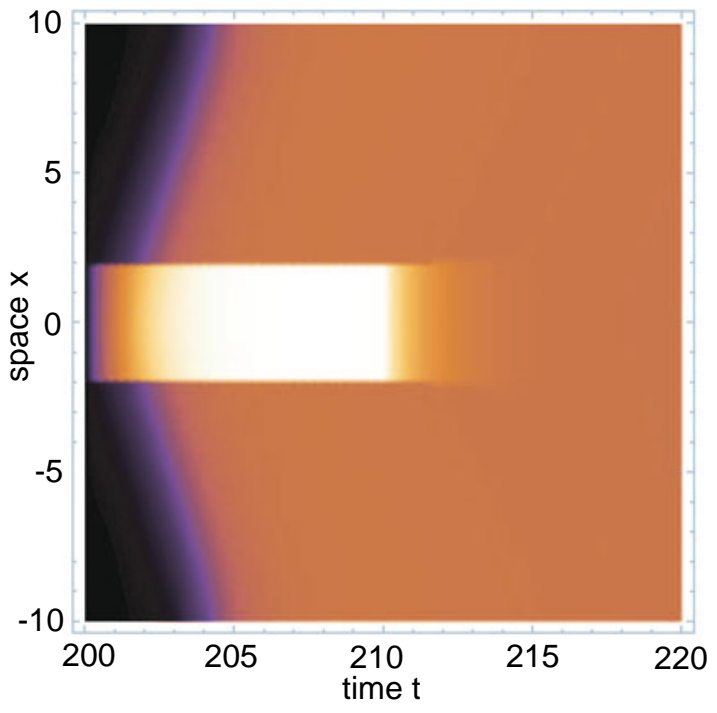

(c)

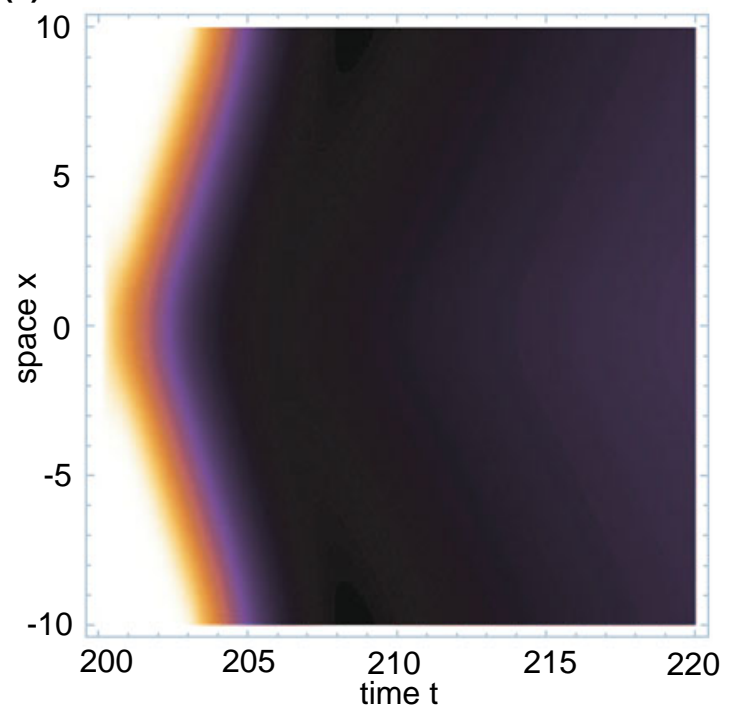

Fig. 7 Same as Fig. 6 except that $\tau_{s}=500$. Onset of trigger stimulus is at $t_{0}=200$. Both the frequency of spontaneous oscillations and the speed of the wave are higher with $c \sim 3$

the zero sets $\operatorname{Re}[E[\lambda]]$ and $\operatorname{Im}[E[\lambda]]$ for the baseline parameter values, we find that there exists only a single eigenvalue (at $\lambda=0$ ), indicating that the traveling front is linearly stable. This result persists for other choices of parameters for which a traveling front exists.

\section{Numerical simulations}

In our analysis of binocular rivalry waves (Section 3), we neglected the dynamics of the depression variables by making an adiabatic approximation. In this section we numerically solve the full system of Eqs. (2.1) and (2.2) and show that traveling fronts persist when the dynamics of the depression variables is included. We begin by considering a single trigger stimulus in the form of a temporary, spatially localized increase in the

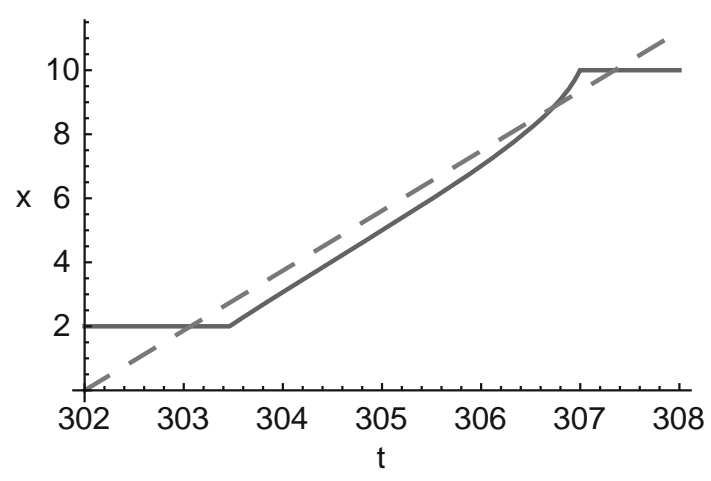

Fig. 8 Plot of the spatial location $x$ vs. time $t$ of the point at which $u(x, t)=0.3$ (solid curve). Same parameter values as Fig. 6 with onset of trigger stimulus at $t_{0}=300$. There is a latency period before the wave front reaches the initial location of the point $u=0.3$. The wave front moves with approximately constant speed that is consistent with the theoretical value as indicated by the dashed line of constant slope 


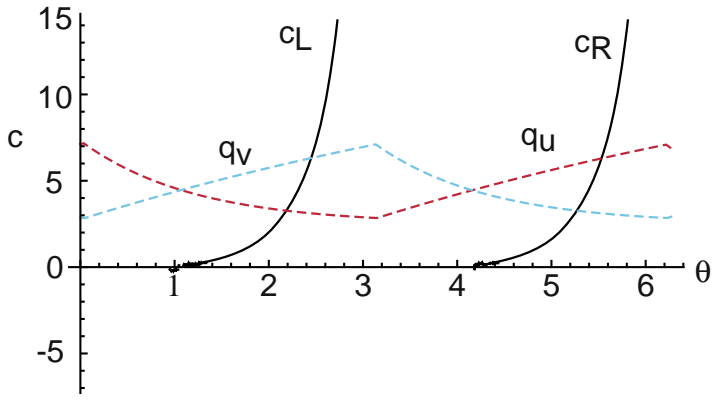

Fig. 9 Wavespeed vs phase (solid curve) along one cycle of spontaneous oscillations. Here $c_{L}\left(c_{R}\right)$ denotes the speed of a wave induced in the suppressed left (right) eye network. Also shown are the temporal profiles of the depression variables (dashed curves). Same parameter values as Fig. 7

input strength to the suppressed eye network, which for concreteness we take to be the left eye. Thus,

$$
\begin{aligned}
& I_{u}(x, t)=H\left(t-t_{0}\right) H\left(\Delta t+t_{0}-t\right) H(\Delta x-|x|) \Delta I+I, \\
& I_{v}(x, t)=I
\end{aligned}
$$

where $H$ is again the Heaviside function. The trigger stimulus is switched on at $t=t_{0}$ and switched off at
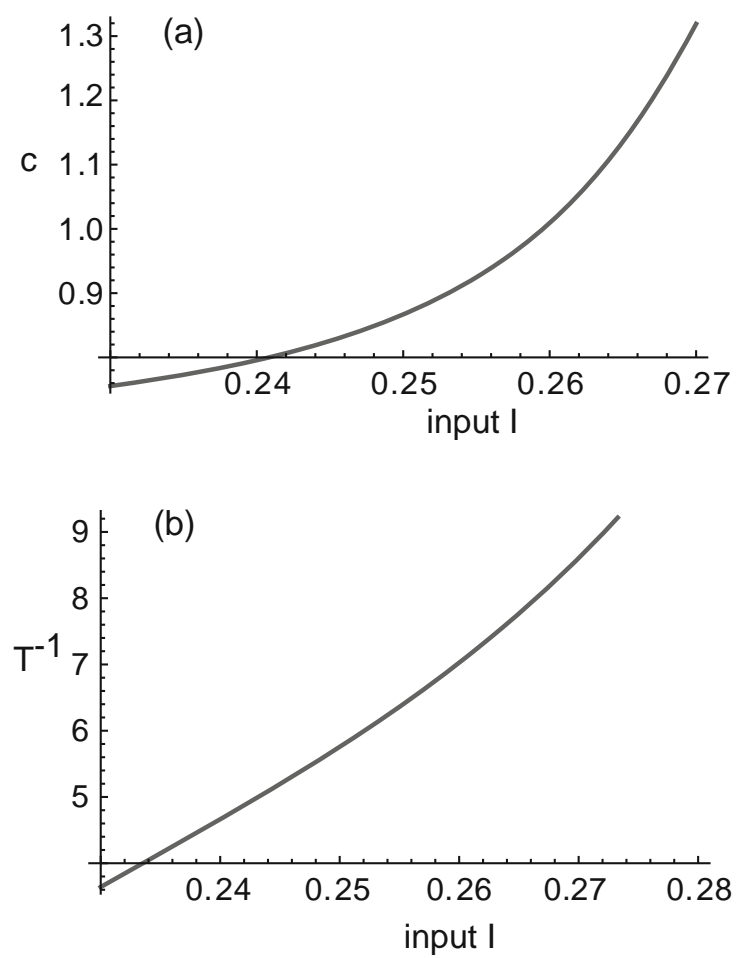

Fig. 10 Wavespeed and frequency of spontaneous oscillations covary. Plot of (a) wavespeed $c$ and (b) alternation rate $\frac{1}{T}$ as a function of stimulus contrast $I$. The depression parameters $Q_{u}, Q_{v}$ are determined by taking the phase $\theta$ of stimulus onset to be $\theta=3 \pi / 2$. All other parameters are given by the baseline values of Fig. 4 time $t=t_{0}+\Delta t$, and consists of an increase in input strength $\Delta I$ in a region of width $2 \Delta x$ centered about $x=0$. In order for traveling waves to be induced in our model, the size $\Delta x$ of the excited region has to be of the order of $\sigma_{e}$, otherwise the effects of the perturbation simply die away. This is consistent with the size of perturbation used in the experiments by Kang et al. (2009, 2010), which was of size 0.2 degrees, corresponding to $0.8 \mathrm{~mm}$ of cortical tissue. Their perturbations were shown for $200 \mathrm{~ms}$, similar to the time interval of $\Delta t=10$ used in our simulations. Two examples of induced binocular rivalry waves are shown in Figs. 6 and 7. In these examples, the wave speed is fast relative to the frequency of spontaneous oscillations so the depression variables change very little over the time during which the wave is traveling. Hence, there is a good match between properties of the numerically simulated wave and our analytical results of Section 3. In Fig. 8 we further illustrate the good agreement between theory
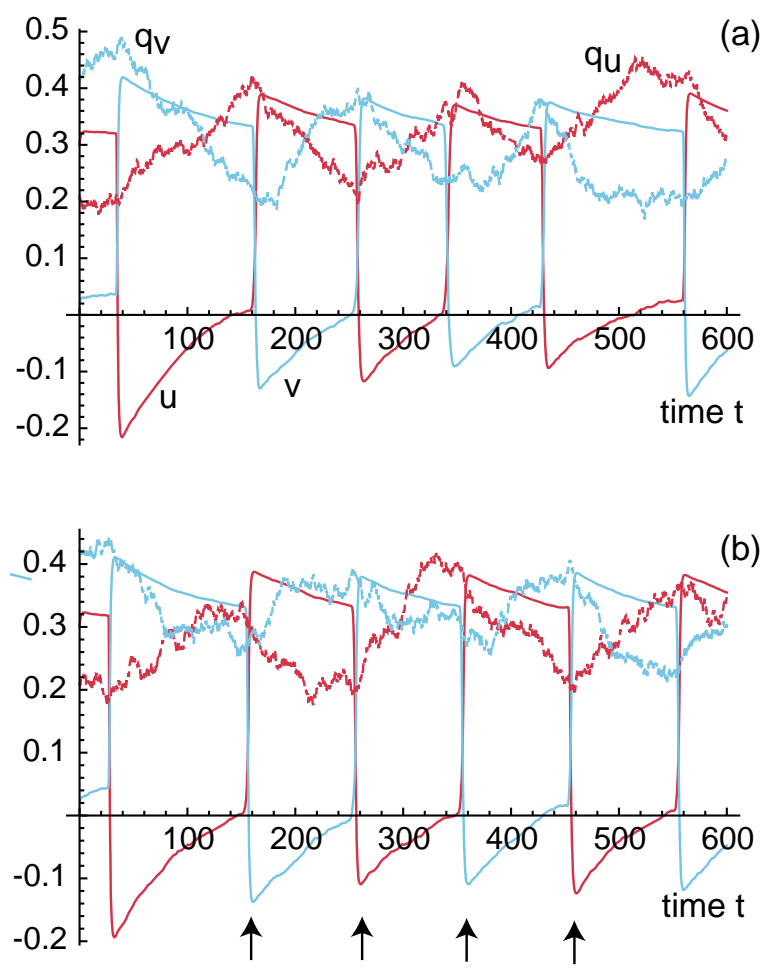

Fig. 11 Comparison of spontaneous and periodically forced binocular rivalry oscillations in the presence of noise. The time evolution of the activity and depression variables are shown at a fixed spatial location $(x=7)$. Noise strength $\sigma=1$ and all other parameter values are as in Fig. 7. Left (right) eye variables are shown in red (blue) with noise in the depression variables. (a) Homogeneous oscillatory solution, which shows some irregularity due to noise in the depression variables. (b) Periodically triggered alternations in dominance. Onset of each trigger stimulus is indicated by an arrow. Initial trigger occurs at $t_{0}=150$ and time between triggers is $T_{0}=100$ 
and numerics by plotting the location $x$ vs. time $t$ of a fixed point in traveling wave coordinates given by $u(x, t)=0.3$. As expected, there is a small variation in wave speed due to the dynamics of the depression variables but the mean speed is consistent with theory.

Since the network operates in a regime where spontaneous rivalry oscillations occur in the absence of any trigger stimuli, it follows from our analytical results (Section 3) that the speed of the induced wave will depend on which phase of the limit cycle the trigger stim- ulus is initiated. That is, let the time of stimulus onset be $t_{0}=n T+\theta T /(2 \pi)$, where $T$ is the period of spontaneous oscillations, $n$ is an integer and $0 \leq \theta<2 \pi$. Denoting the oscillatory solution of the depression variables on the limit cycle by $\left\{q_{u}^{*}(\phi), q_{v}^{*}(\phi) \mid 0 \leq \phi<2 \pi\right\}$, we set $Q_{u}=q_{u}^{*}(\theta), Q_{v}=q_{v}^{*}(\theta)$ where $Q_{u}, Q_{v}$ are the fixed values appearing in Eqs. (3.18) and (3.19) under the adiabatic approximation. It follows that the wave speed calculated from Eq. (3.29) is phase-dependent: $c=c\left(Q_{u}, Q_{v}\right)=c(\theta)$. An example plot of wave speed

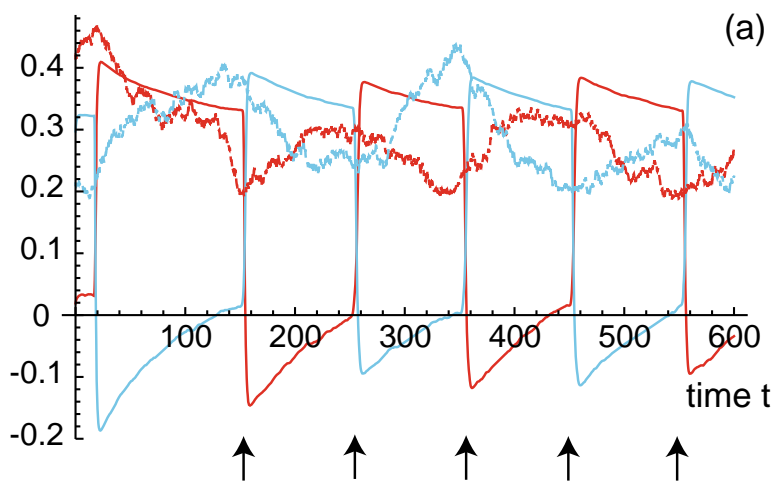

(b)
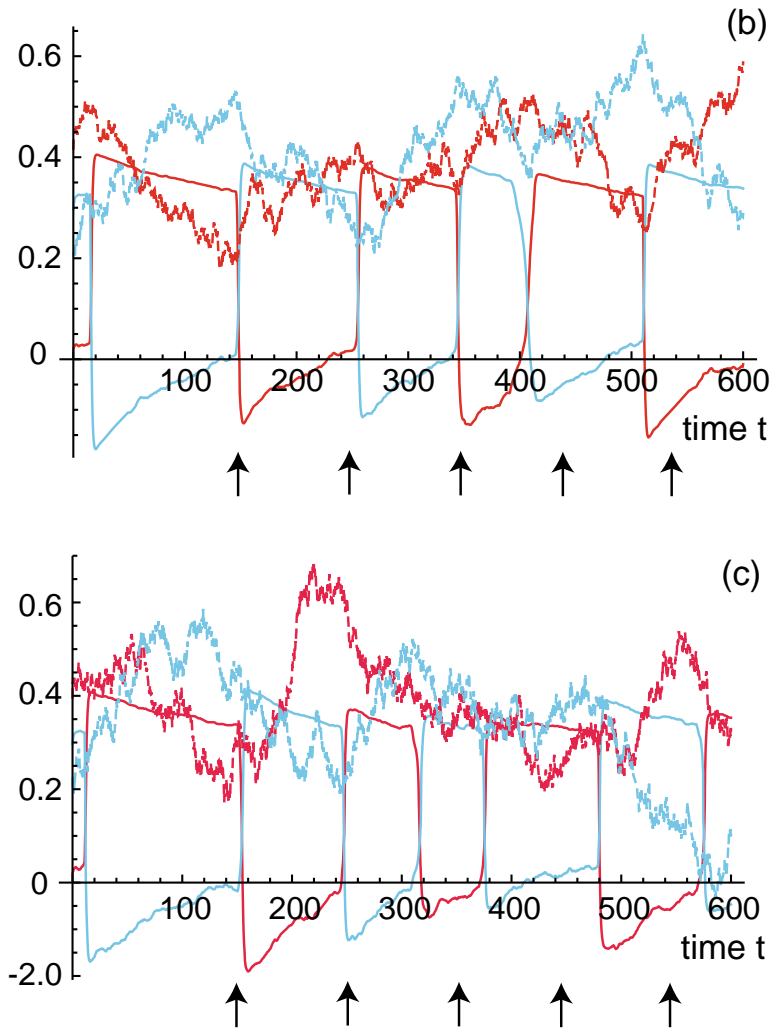

(d)

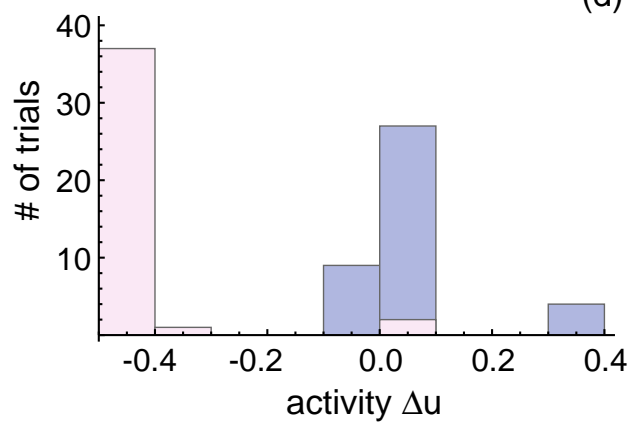

(e)

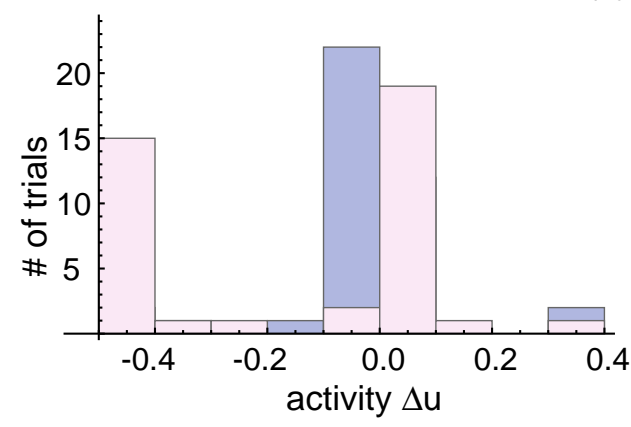

(f)

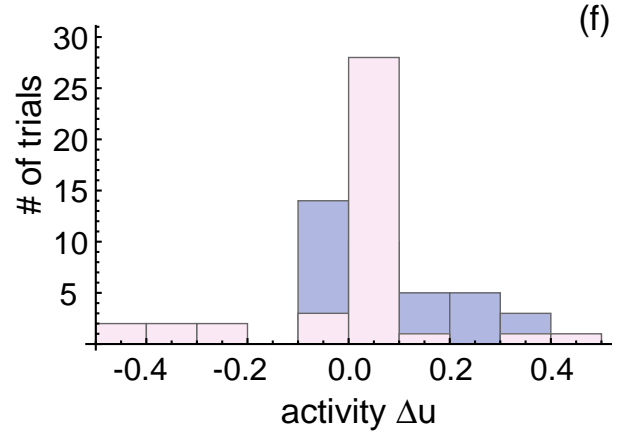

Fig. 12 Breakdown of mode-locking as noise strength increases. $(\mathbf{a}-\mathbf{c})$ The time evolution of the activity and depression variables are shown at a fixed spatial location $(x=7)$ for various noise strengths: (a) $\sigma=1$, (b) $\sigma=2,(\mathbf{c}) \sigma=3$. All other parameter values are as in Fig. 11. Onset of each trigger stimulus is indicated by an arrow. Initial trigger occurs at $t_{0}=150$ and time between triggers is $T_{0}=100$. (d-f) Corresponding histograms showing how the change in activity $\Delta u=u\left(x_{0}, t_{1}\right)-u\left(x_{0}, t_{2}\right)$ at a fixed location $x_{0}=7$ and two different times $\left(t_{1}=450, t_{2}=470\right)$ is distributed over multiple trials. Pink (purple) bars indicate distribution in the presence (absence) of periodic trigger stimuli 
vs phase $\theta$ is shown in Fig. 9. We see that a rivalry wave only exists if the trigger stimulus occurs in the latter $\sim \frac{2}{3}$ of the half-cycle, with faster waves induced closer to the point of spontaneous switching. Now suppose that we keep the phase of the trigger stimulus fixed and determine how both the natural frequency $1 / T$ and wave speed $c$ vary with stimulus contrast $I$. The results are presented in Fig. 10. The wave speed is determined from Eq. (3.29), whereas the period $T$ is calculated using the analytical results of Kilpatrick and Bressloff (2010). It can be seen that faster natural alternation
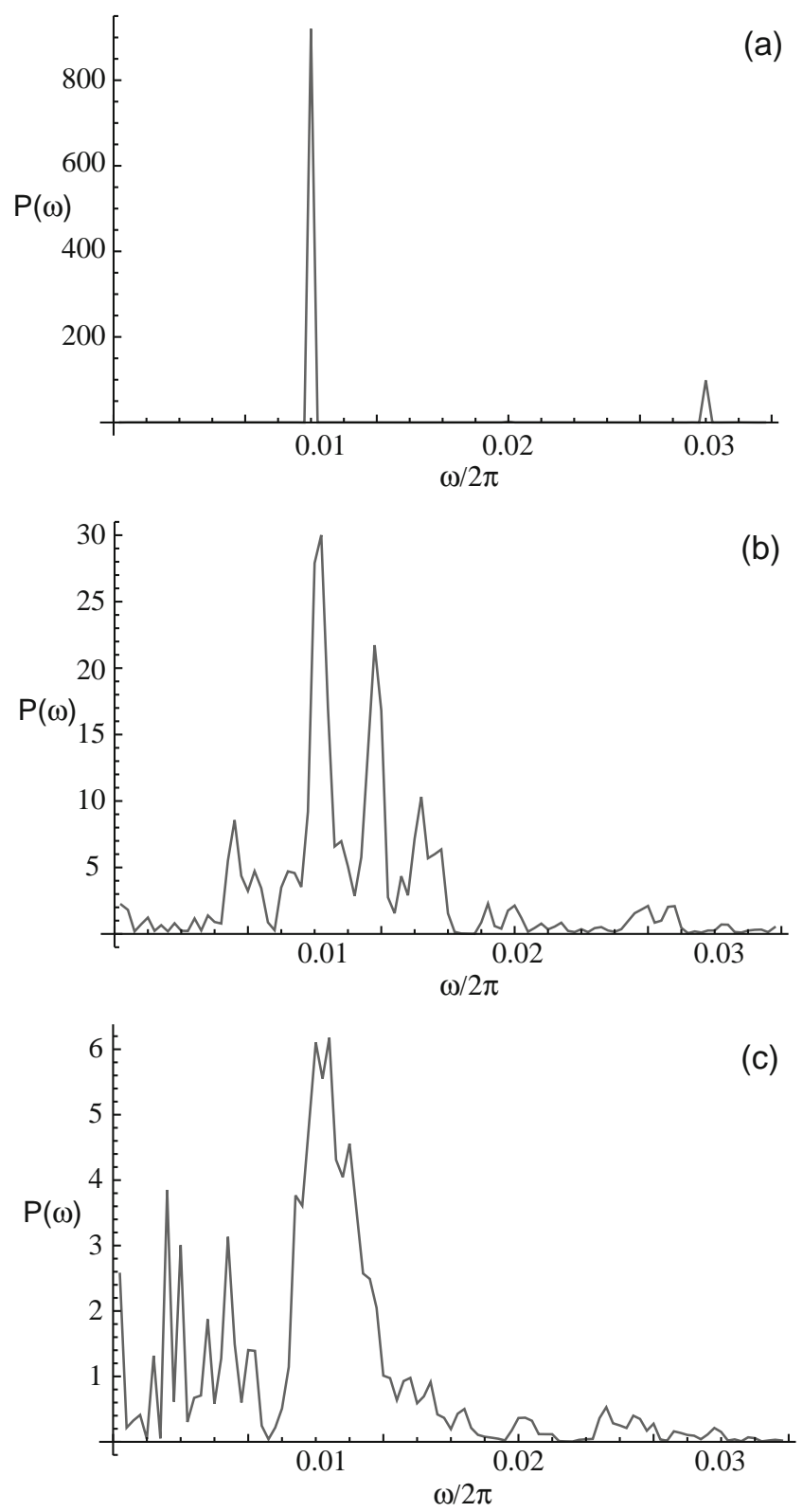

Fig. 13 Power spectrum $P(\omega)=|u(\omega)|^{2}$ where $u(\omega)$ is the Fourier transform of time varying signal $u\left(x_{0}, t\right)$ for $x_{0}=7$ : (a) $\sigma=1,(\mathbf{b}) \sigma=2,(\mathbf{c}) \sigma=3$. All other parameters as in Fig. 12 rates lead to faster wave speeds. (Wavespeed and frequency also covary with changes in the depression rate constant $\beta$, for example). Interestingly, an analogous result was obtained by Kang et al. (2009, 2010), who found experimentally that traveling waves were faster for subjects whose natural rate of binocular rivalry oscillations was higher. However, as noted in Section 3, the wave speed was averaged over several cycles of trigger stimuli in the psychophysical experiments. Given the stochastic nature of spontaneous oscillations, we expect such averaging to reduce the dependence on the phase $\theta$.

In order to relate our model more closely with the experimental protocol of Kang et al. (2009, 2010), we have also carried out simulations of the full model (2.1) and (2.2) in the presence of additive noise and periodic trigger stimuli. For the sake of illustration, we introduce spatially uncorrelated additive white noise terms to the the dynamical equations for the depression variables according to

$$
\begin{aligned}
\tau_{s} \frac{\partial q_{u}(x, t)}{\partial t}= & 1-q_{u}(x, t)-\beta q_{u}(x, t) f(u(x, t)) \\
& +\sigma \xi_{u}(x, t) \\
\tau_{s} \frac{\partial q_{v}(x, t)}{\partial t}= & 1-q_{v}(x, t)-\beta q_{v}(x, t) f(v(x, t)) \\
& +\sigma \xi_{v}(x, t)
\end{aligned}
$$

with

$$
\left\langle\xi_{u}(x, t)\right\rangle=\left\langle\xi_{v}(x, t)\right\rangle=0
$$
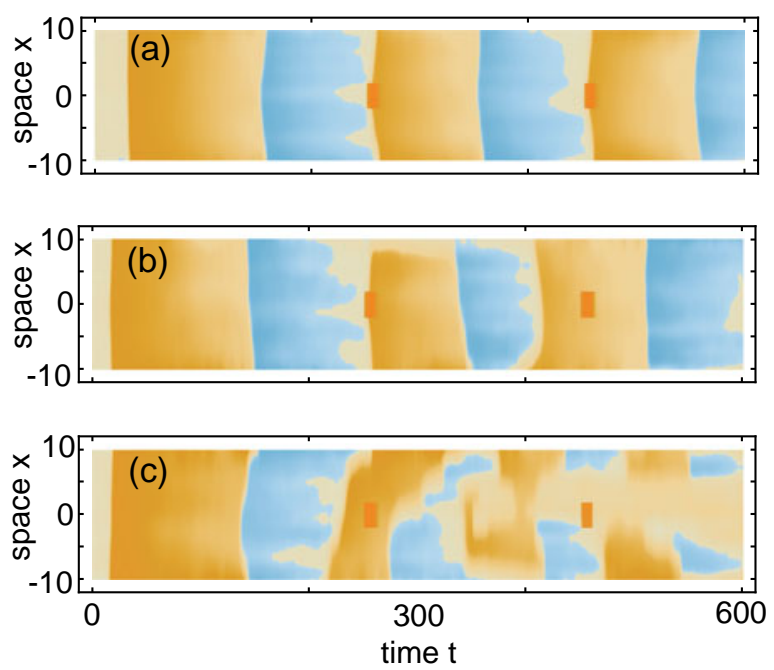

Fig. 14 Space-time plots of periodically triggered binocular rivalry waves in left eye network for increasing levels of noise: (a) $\sigma=1,(\mathbf{b}) \sigma=2,(\mathbf{c}) \sigma=3$. Red rectangles indicate duration and spatial extent of trigger stimuli. All other parameters as in Fig. 12 
(a)

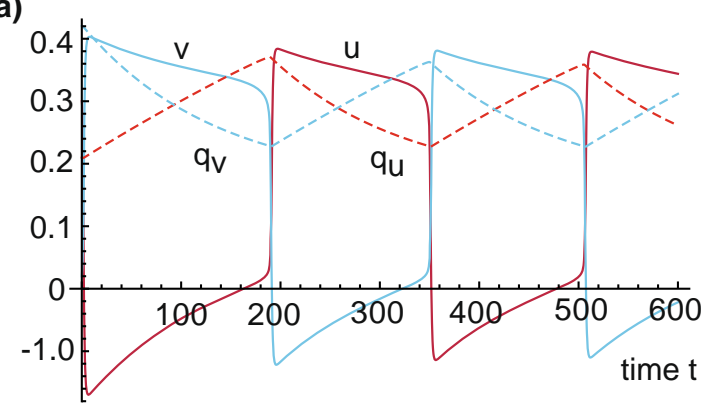

(b)

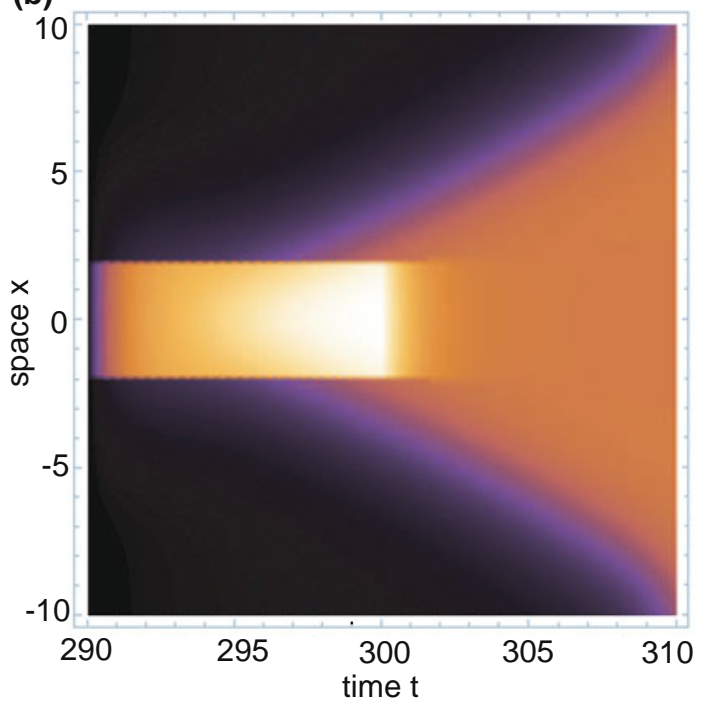

Fig. 15 Induction of a solitary binocular rivalry wave in the case of a smooth sigmoidal firing rate function. Gain of sigmoid is $\eta=80$. Other parameter values are as in Fig. 6. (a) Homogeneous oscillatory solution in which there is spontaneous periodic switching between left and right eye dominance. Plot against time of the left eye neural activity $u$ (solid red), the right eye neural activity $v$ (solid blue) together with the corresponding depression variables $q_{u}$ (dashed red) and $q_{v}$ (dashed blue).

and

$$
\begin{aligned}
& \left\langle\xi_{u}(x, t) \xi_{u}\left(x^{\prime}, t^{\prime}\right)\right\rangle=\delta\left(x-x^{\prime}\right) \delta\left(t-t^{\prime}\right), \\
& \left\langle\xi_{v}(x, t) \xi_{v}\left(x^{\prime}, t^{\prime}\right)\right\rangle=\delta\left(x-x^{\prime}\right) \delta\left(t-t^{\prime}\right) \\
& \left\langle\xi_{u}(x, t) \xi_{v}\left(x^{\prime}, t^{\prime}\right)\right\rangle=0 .
\end{aligned}
$$

Furthermore, the inputs $I_{u}$ and $I_{v}$ in Eqs. (2.1a) and (2.2a) take the form

$$
\begin{aligned}
I_{u}(x, t)= & I+\sum_{m=0,2,4, \ldots} H\left(t-t_{0}-m T_{0}\right) \\
& \times H\left(\Delta t+t_{0}+m T_{0}-t\right) H(\Delta x-|x|) \Delta I, \\
I_{v}(x, t)= & I+\sum_{m=1,3,5, \ldots} H\left(t-t_{0}-m T_{0}\right) \\
& \times H\left(\Delta t+t_{0}+m T_{0}-t\right) H(\Delta x-|x|) \Delta I
\end{aligned}
$$

(d)

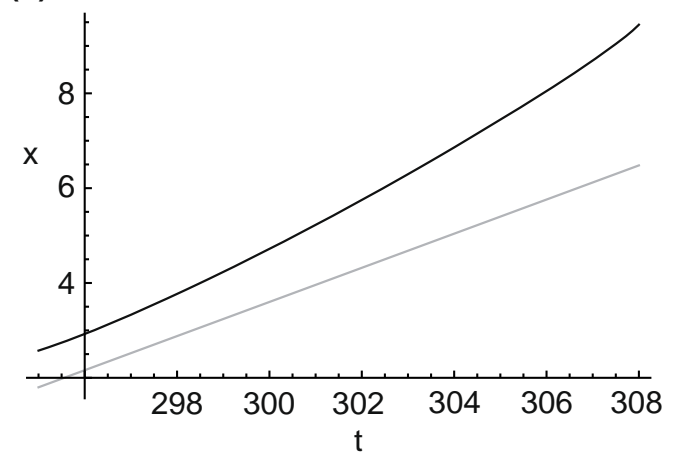

(c)

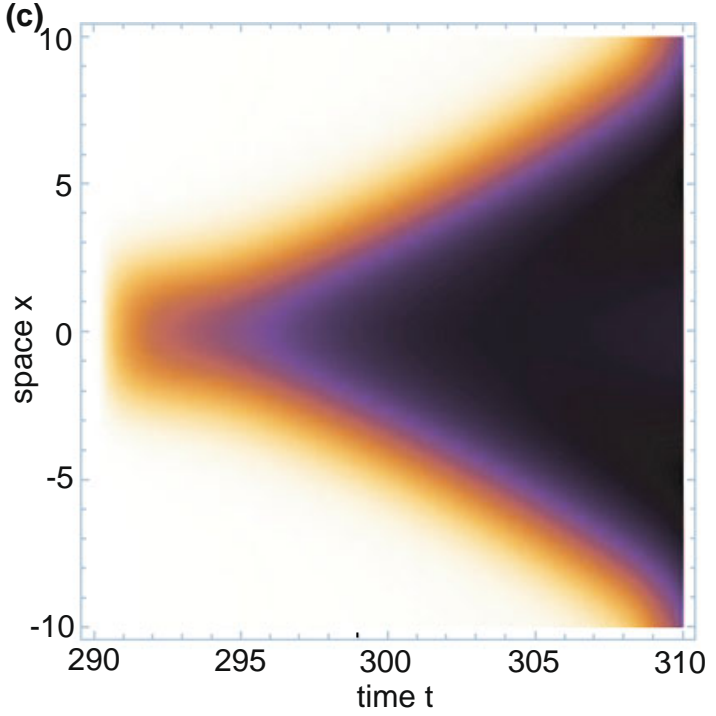

(b,c) Space-time plots of $u(x, t)$ and $v(x, t)$ following onset of the trigger stimulus to the left eye at time $t_{0}=290$, which involves a temporary increase in the input strength $I_{u}$ from 0.24 to 0.74 within the domain $-2 \leq x \leq 2$. Lighter (darker) colors indicate higher (lower) activity values. (d) Plot of the spatial location $x$ vs. time $t$ of the point at which $u(x, t)=0.2$ (black curve). The speed calculated for the Heaviside case is given by the slope of the straight line (grey curve)

That is, starting at time $t=t_{0}$, the left and right eye networks receive a $T_{0}$-periodic alternating sequence of trigger stimuli, each of which consists of an enhancement of input strength in the domain $|x|<\Delta x$ that has duration $\Delta t$.

Results from numerical simulations of the full model given by Eqs. (2.1a), (2.2a), (4.1) and (4.2) are shown in Figs. 11 and 12. It can be seen that if the noise strength $\sigma$ is not too large then there is reliable switching between left and right eye dominance that phase locks to the periodic trigger stimuli. However, as $\sigma$ increases to a value of around $\sigma \approx 2$, phase locking starts to break down. As a further illustration of how modelocking depends on the level of noise, we also plot in Fig. 12 histograms comparing the spontaneous and forced distributions of activity $\Delta u=u\left(x_{0}, t_{1}\right)-u\left(x_{0}, t_{2}\right)$ 
at a fixed location $x=x_{0}$ and fixed times time $t_{1}, t_{2}$ over multiple trials (simulation runs). For a noise strength $\sigma=1$ it can be seen that mode locking causes a very strong tendency to be in the completely opposite state to that without mode locking, whereas for higher noise strengths $\sigma=2,3$ the distribution is much wider and in some situations the forcing did not change the state from the unforced case (breakdown of mode-locking). The corresponding power spectrum of the time varying signal $u\left(x_{0}, t\right)$ is plotted in Fig. 13, which shows a broadening of the peak at the forcing frequency $\omega / 2 \pi=$ $1 / T_{0}=0.01$ as the noise strength increases. Finally, the disruption of binocular rivalry waves with increasing noise is illustrated by the space-time plots of the left network activity variable $u(x, t)$ in Fig. 14 .

So far we have taken the firing rate function to be the Heaviside function (2.4) in order to compare our numerics with the analysis of Section 3. As in many previous studies of neural field equations dating back to Amari (1977), the use of Heavisides allowed us to construct explicit traveling wave solutions and to derive an explicit formula for the speed of the wave. However, it is important to check that the same basic mechanism of wave induction and propagation carries over to more realistic sigmoidal rate functions. This is indeed found to be the case, as is illustrated in Fig. 15. The speed of the wave is more variable than the Heaviside case but is still comparable in magnitude to theoretical predictions.

Note on simulations All deterministic simulations were carried out by discretizing space and solving the resulting system of ODEs with Mathematica 8.0's ODE solver (NDSolve). Discretization of the neural field model with additive noise generated a system of stochastic ODEs, which were then simulated in $\mathrm{C}++$ code (with boost and Intel TBB) using the Euler-Maruyama method.

\section{Discussion}

In this paper we analyzed a neural field model of binocular rivalry waves in primary visual cortex. Formulating the problem in terms of continuum neural field equations allowed us to study the short time behavior associated with the propagation of eye dominance from an analytical perspective. In particular, we established that in order for traveling waves to exist, a symmetry breaking mechanism needs to be present. That is, the equations for the left and right eye networks have to be different on the time scales during which traveling waves propagate. We identified one possible mecha- nism for providing the necessary symmetry breaking, namely, synaptic depression. However, we expect that other forms of slow adaptation such as spike frequency adaptation would also work. We then showed analytically that for plausible values of biological parameters such as the range of synaptic interactions and the membrane time constant, we could recover wave speeds similar to those seen in experiments (Wilson et al. 2001; Kang et al. 2009, 2010). Moreover, our model exhibited the expected dependence of wave speed on parameters that can be experimentally varied, such as stimulus contrast. It also replicated the experimental finding that wave speed covaries with the natural frequency of spontaneous rivalry oscillations. Finally, numerical simulations of the full system were in good agreement with our analytical short time scale results in the case of a Heaviside firing rate function. We also found that binocular rivalry waves persisted in the case of smooth sigmoid firing rate functions and in the presence of additive white noise in the depression variable dynamics. However, mode-locking to periodic trigger stimuli broke down when the noise strength became too large.

In future work it would be interesting to extend our neural field model to a more realistic network topology. Instead of considering one line of neurons for each eye, we could consider a circle of neurons for each point in the visual field-this would allow us to take orientation information into account using an extension of the coupled ring model (Bressloff et al. 2001; Bressloff and Cowan 2002). We could then investigate the experimental observation that the speed of binocular rivalry waves depends on the orientation of the left and right eye grating stimuli (Wilson et al. 2001). Another important issue that we only partially addressed in this paper is the role of noise. A number of recent studies have considered stochastic models of binocular rivalry and the statistics of dominance times, but have neglected spatial effects (Moreno-Bote et al. 2007; Shpiro et al. 2009; Braun and Mattia 2010). One particularly interesting implication of our work is that purely noise-driven switching between rivalrous states in the absence of adaptation cannot by itself generate rivalry waves. That is, if we were to include zero mean, spatially uncorrelated additive noise in the activity equations (3.1) and (3.2) without any adaptation, then this would not be sufficient to break the exchange symmetry of the left and right eye networks. Hence, if noise is inadequate as a symmetry-breaking mechanism, this would argue against studies that favor a noise-only (or at least noise-dominated) approach to modeling rivalry (Moreno-Bote et al. 2007; Gigante et al. 2009). Developing a further understanding of the combined effects of adaptation, noise and wave 
propagation on periodically triggered switching would allow us to address relevant issues such as determining the stimulus trigger period in a noisy system that gives the highest probability of a dominance switch. This would add to the results already found in this paper (such as wave speed variation with phase angle) that could be experimentally tested and lead to further refinements of the neural field model.

\section{References}

Abbott, L. F., Varela, J. A., Sen, K., \& Nelson, S. B. (1997). Synaptic depression and cortical gain control. Science, 275, 220-224.

Amari, S. (1977). Dynamics of pattern formation in lateralinhibition type neural fields. Biological Cybernetics, 27, 7787.

Angelucci, A., Levitt, J. B., Walton, E. J. S., Hupe, J.-M., Bullier, J., \& Lund, J. S. (2002). Circuits for local and global signal integration in primary visual cortex. Journal of Neuroscience, $22,8633-8646$.

Bart, E., Bao, S., \& Holcman, D. (2005). Modeling the spontaneous activity of the auditory cortex.. Journal of Computational Neuroscience, 19, 357-378.

Ben-Yishai, R., Bar-Or, R. L., \& Sompolinsky, H. (1995). Theory of orientation tuning in visual cortex. Proceedings of the National Academy of Sciences of the United States of America, 92, 3844-3848.

Blake, R. (2001). A primer on binocular rivalry, including current controversies. Brain and Mind, 2, 5-38.

Blake, R., \& Logothetis, N. (2002). Visual competition. Nature Reviews Neuroscience, 3, 1-11.

Blasdel, G. G. (1992). Orientation selectivity, preference, and continuity in monkey striate cortex. Journal of Neuroscience, 12, 3139-3161.

Braun, J. C., \& Mattia, M. (2010). Attractors and noise: Twin drivers of decisions and multistability. Neuroimage, 52, 740751.

Bressloff, P. C., \& Cowan, J. D. (2002). An amplitude equation approach to contextual effects in visual cortex. Neural Computation, 14, 493-525.

Bressloff, P. C., Cowan, J. D., Golubitsky, M., Thomas, P. J., \& Wiener, M. (2001). Geometric visual hallucinations, Euclidean symmetry and the functional architecture of striate cortex. Philosophical Transactions of the Royal Society B: Biological Sciences, 40, 299-330.

Chance, F. S., Nelson, S. B., \& Abbott, L. F. (1998). Synaptic depression and the temporal response characteristics of V1 cells. Journal of Neuroscience, 18, 4785-4799.

Coombes, S., \& Owen, M. R. (2004). Evans functions for integral neural field equations with Heaviside firing rate function. SIAM Journal on Applied Dynamical Systems, 3, 574-600.

De Weerd, P., Desimone, R., \& Ungerleider, L. G. (1998). Perceptual filling in: A parametric study. Vision Research, 38, 2721-2734.

Ferster, D., \& Miller, K. D. (2000). Neural mechanisms of orientation selectivity in the visual cortex. Annual Review of Neuroscience, 23, 441-471.

Fox, R., \& Rasche, F. (1969). Binocular rivalry and reciprocal inhibition. Perception \& Psychophysics, 5, 215-217.

Gigante, G., Mattia, M., Braun, J., \& Del Giudice, P. (2009). Bistable perception modeled as competing stochastic inte- grations at two levels. PLoS Computational Biological 5(7), e1000430.

Gold, J. M., \& Shubel, E. (2006). The spatiotemporal properties of visual completion measured by response classification. Journal of Vision, 6, 356-365.

Hadjikhani, N., Sanchez Del Rio, M., Wu, O., Schwartz, D., Bakker, D., et al. (2001). Mechanisms of migraine aura revealed by functional MRI in human visual cortex. Proceedings of the National Academy of Sciences of the United States of America, 98, 4687-4692.

Hubel, D. H., \& Wiesel, T. N. (1962). Receptive fields, binocular interaction and functional architecture in the cat's visual cortex. Journal of Physiology, 160, 106-154.

Hubel, D. H., \& Wiesel, T. N. (1977). Functional architecture of macaque monkey visual cortex. Proceedings of the Royal Society of London. Series B, Biological Sciences, 198, 1-59.

Jancke, D., Chavane, F., Maaman, S., \& Grinvald, A. (2004). Imaging cortical correlates of illusion in early visual cortex. Nature, 428, 423-426.

Kang, M., Heeger, D. J., \& Blake, R. (2009). Periodic perturbations producing phase-locked fluctuations in visual perception. Journal of Vision, 9(2:8), 1-12.

Kang, M., Lee, S.-H., Kim, J., Heeger, D. J., \& Blake, R. (2010). Modulation of spatiotemporal dynamics of binocular rivalry by collinear facilitation and pattern-dependent adaptation. Journal of Vision, 10(11:3), 1-15.

Katz, L. C., Gilbert, C. D., \& Wiesel, T. N. (1989). Local circuits and ocular dominance columns in monkey striate cortex. Journal of Neuroscience, 9, 1389-1399.

Kilpatrick, Z. P., \& Bressloff, P. C. (2010). Binocular rivalry in a competitive neural network with synaptic depression. SIAM Journal of Applied Dynamical Systems, 9, 1303-1347.

Laing, C. R., \& Chow, C. C. (2002). A spiking neuron model for binocular rivalry. Journal of Computational Neuroscience, $12,39-53$

Lee, S.-H., Blake, R., \& and Heeger, D. J. (2005). Traveling waves of activity in primary visual cortex during binocular rivalry. Nature Neuroscience, 8, 22-23.

Loxley, P. N., \& Robinson, P. A. (2009). Soliton model of competitive neural dynamics during binocular rivalry. Physical Review Letters, 102, 258701.

Lund, J. S., Angelucci, A., \& Bressloff, P. C. (2003). Anatomical substrates for functional columns in macaque monkey primary visual cortex. Cerebral Cortex, 12, 15-24.

Moreno-Bote, R., Rinzel, J., \& Rubin, N. (2007). Noise-induced alternations in an attractor network model of perceptual bistability. Journal of Neurophysiology, 98, 1125-1139.

Pinto, D. J., \& Ermentrout, G. B. (2001). Spatially structured activity in synaptically coupled neuronal networks: I. Traveling fronts and pulses. SIAM Journal of Applied Mathematics, 62, 206-228.

Sandstede, B. (2007). Evans functions and nonlinear stability of traveling waves in neuronal network models. International Journal of Bifurcation and Chaos in Applied Sciences and Engineering, 17, 2693-2704.

Shpiro, A., Curtu, R., Rinzel, J., \& Rubin, N. (2007). Dynamical characteristics common to neuronal competition models. Journal of Neurophysiology, 97, 462-473.

Shpiro, A., Moreno-Bote, R., Rubin, N. R., \& Rinzel, J. (2009). Balance between noise and adaptation in competition models of perceptual bistability. Journal of Computational Neuroscience, 27, 37-54.

Skinner, F. K., Kopell, N., \& Marder, E. (1994). Mechanisms for oscillation and frequency control in reciprocally inhibitory model neural networks. Journal of Computational Neuroscience, 1, 69-87. 
Sincich, L., \& Blasdel, G. G. (2001). Oriented axon projections in primary visual cortex of the monkey. Journal of Neuroscience, 21, 4416-4426.

Sterzer, P., Kleinschmidt, A., \& Rees, G. (2009). The neural bases of multistable perception. Trends in Cognitive Sciences, 13, 310-318.

Tabak, J., Senn, W., O’Donovan, M. J., \& Rinzel, J. (2000). Modeling of spontaneous activity in developing spinal cord using activity-dependent depression in an excitatory network. Journal of Neuroscience, 20, 3041-3056.

Taylor, A. L., Cottrell, G. W., \& Kristan, W. B. (2002). Analysis of oscillations in a reciprocally inhibitory network with synaptic depression. Neural Computation, 14, 561-581.

Tootell, R. B., Switkes, E., Silverman, M. S., \& Hamilton, S. L. (1988). Functional anatomy of macaque striate cortex. II.
Retinotopic organization. Journal of Neuroscience, 8, 15311568.

Tsodyks, M., Pawelzik, K., \& Markram, H. (1998). Neural networks with dynamic synapses. Neural Computation, 10, 821835.

Varela, J. A., Sen, K., Gibson, J., Fost, J., Abbott, L. F., \& Nelson, S. B. (1997). A quantitative description of shortterm plasticity at excitatory synapses in layer $2 / 3$ of rat primary visual cortex. Journal of Neuroscience, 17, 79267940.

Wilson, H. R., Blake, R., \& Lee, S. H. (2001). Dynamics of traveling waves in visual perception. Nature, 412, 907-910.

Zhang, L. (2003). On stability of traveling wave solutions in synaptically coupled neuronal networks. Differential and Integral Equations, 16, 513-536. 